\title{
ON THE CLASSIFICATION OF POINTED FUSION CATEGORIES UP TO WEAK MORITA EQUIVALENCE
}

\author{
BERNARDO URIBE
}

\begin{abstract}
A pointed fusion category is a rigid tensor category with finitely many isomorphism classes of simple objects which moreover are invertible. Two tensor categories $\mathcal{C}$ and $\mathcal{D}$ are weakly Morita equivalent if there exists an indecomposable right module category $\mathcal{M}$ over $\mathcal{C}$ such that $\operatorname{Fun}_{\mathcal{C}}(\mathcal{M}, \mathcal{M})$ and $\mathcal{D}$ are tensor equivalent. We use the Lyndon-Hochschild-Serre spectral sequence associated to abelian group extensions to give necessary and sufficient conditions in terms of cohomology classes for two pointed fusion categories to be weakly Morita equivalent. This result may permit to classify the equivalence classes of pointed fusion categories of any given global dimension.
\end{abstract}

\section{INTRODUCTION}

Pointed fusion categories are rigid tensor categories with finitely many isomorphism classes of simple objects with the property that all simple objects are invertible. Any pointed fusion category $\mathcal{C}$ is equivalent to the fusion category $\operatorname{Vect}(G, \omega)$ of complex vector spaces graded by the finite group $G$ together with the associativity constraint defined by the 3 -cocycle $\omega \in Z^{3}\left(G, \mathbb{C}^{*}\right)$. Whenever we have a right module category $\mathcal{M}$ over $\mathcal{C}$ we can define the dual category $\mathcal{C}_{\mathcal{M}}^{*}:=\operatorname{Fun}_{\mathcal{C}}(\mathcal{M}, \mathcal{M})$ which becomes a tensor category via composition of functors. Whenever $\mathcal{C}$ is a fusion category and $\mathcal{M}$ is an indecomposable fusion category, the dual category $\mathcal{C}_{\mathcal{M}}^{*}$ is also a fusion category [11, $\left.\S 2.2\right]$. An indecomposable module category $\mathcal{M}$ of $\operatorname{Vect}(G, \omega)$ may be defined by $\mathcal{M}=\mathcal{M}(K, \mu)$ where $K$ is the space of cosets $K:=A \backslash G$ for $A$ a subgroup of $G$ and $\mu \in C^{2}\left(G, \operatorname{Map}\left(K, \mathbb{C}^{*}\right)\right)$ is a cochain that satisfies the equation $\delta_{G} \mu^{-1}=\omega$. Two tensor categories $\mathcal{C}$ and $\mathcal{D}$ are weakly Morita equivalent if there exists an indecomposable right module category $\mathcal{M}$ over $\mathcal{C}$ such that $\mathcal{C}_{\mathcal{M}}^{*}$ and $\mathcal{D}$ are tensor equivalent $[9$, Def 4.2$]$.

Now, if we have two pointed fusion categories $\operatorname{Vect}(G, \omega)$ and $V e c t(\widehat{G}, \widehat{\omega})$, what are the necessary and sufficient conditions for them to be weakly Morita equivalent? This question was raised in [3, 8], it was answered by Davydov [3, Cor. 6.2] for the case on which both $\omega$ and $\widehat{\omega}$ were trivial, and the general case was answered by Naidu in [10, Theorem 5.8] in terms of the properties that $A, \omega$ and $\mu$ need to satisfy. Nevertheless these conditions were given in equations that a priori had no interpretation in terms of known cohomology classes.

2010 Mathematics Subject Classification. (primary) 18D10, (secondary) 20J06.

Key words and phrases. Tensor Category, Pointed Tensor Category, Weak Morita Equivalence, Fusion Category.

The author acknowledges the financial support of the Max Planck Institute of Mathematics in Bonn, Germany, and of COLCIENCIAS through grant number FP44842-617-2014 of the Fondo Nacional de Financiamiento para la Ciencia, la Tecnología y la Inovación. 
We continue the work started by Naidu in [10] and frame all the calculations done there in the language of the double complex associated to an abelian group extension which induces the Lyndon-Hochschild-Serre spectral sequence. By doing so we are able to obtain in Corollary 3.2 cohomological conditions on $\omega$ in order for the tensor category $\operatorname{Vect}(G, \omega)_{\mathcal{M}(A \backslash G, \mu)}^{*}$ to be pointed, namely that $\omega$ must be cohomologous to a cocycle appearing in $C^{2,1} \oplus C^{3,0}$ of the double complex which induces the Lyndon-Hochschild-Serre spectral sequence associated to the extension $1 \rightarrow A \rightarrow G \rightarrow K \rightarrow 1$.

With the previous result at hand, we construct explicit representatives of $\omega$ and $\mu$ in terms of coordinates and we determine explicitly the groups $\widehat{G}$ and the cocycles $\widehat{\omega}$. The main result of this paper is Theorem 3.9 in which we give the necessary and sufficient conditions for the categories $V e c t(H, \eta)$ and $V e c t(\widehat{H}, \widehat{\eta})$ to be weakly Morita equivalent. We may summarize the conditions as follows: $\operatorname{Vect}(H, \eta)$ and $\operatorname{Vect}(\widehat{H}, \widehat{\eta})$ are weakly Morita equivalent if and only if there exist isomorphisms of groups $\phi: A \rtimes_{F} K \stackrel{\cong}{\rightrightarrows} H$ and $\widehat{\phi}: K \ltimes_{\widehat{F}} \mathbb{A} \cong \stackrel{\cong}{\rightrightarrows} \widehat{H}$ for some finite group $K$ acting on the abelian group $A$, with $F \in Z^{2}(K, A)$ and $\widehat{F} \in Z^{2}(K, \mathbb{A})$ where $\mathbb{A}:=\operatorname{Hom}\left(A, \mathbb{C}^{*}\right)$, such that both $[\widehat{F}]$ and $[F]$ survive respectively the LHS spectral sequence for the groups $A \rtimes_{F} K$ and $K \ltimes_{\widehat{F}} \mathbb{A}$, and such that $\phi^{*} \eta$ is cohomologous to

$$
\omega\left(\left(a_{1}, k_{1}\right),\left(a_{2}, k_{2}\right),\left(a_{3}, k_{3}\right)\right):=\widehat{F}\left(k_{1}, k_{2}\right)\left(a_{3}\right) \epsilon\left(k_{1}, k_{2}, k_{3}\right)
$$

and $\widehat{\phi}^{*} \widehat{\eta}$ is cohomologous to

$$
\widehat{\omega}\left(\left(k_{1}, \rho_{1}\right),\left(k_{2}, \rho_{2}\right),\left(k_{3}, \rho_{3}\right)\right):=\epsilon\left(k_{1}, k_{2}, k_{3}\right) \rho_{1}\left(F\left(k_{2}, k_{3}\right)\right)
$$

where $\epsilon: K^{3} \rightarrow \mathbb{C}^{*}$ satisfies $\delta_{K} \epsilon=\widehat{F} \wedge F$.

Theorem [3.9] may be used to determine the weak Morita equivalence classes of pointed fusion categories of a given global dimension but the cohomological calculations can become very elaborate and are beyond the scope of this article. Nevertheless in section 4 we include a calculation on which we show how Theorem 3.9 can be used to show that there are only seven weak Morita equivalence classes of pointed fusion categories of global dimension four, and in order to calculate the pointed fusion categories which are weakly Morita equivalent to $\operatorname{Vect}\left(Q_{8}, \eta\right)$ for the quaternion group $Q_{8}$.

\section{Preliminaries}

1.1. Abelian group extensions. Consider the short exact sequence of finite groups

$$
1 \longrightarrow A \longrightarrow G \longrightarrow K \longrightarrow 1
$$

with $A$ abelian. Consider $u: K \rightarrow G$ any section of the projection map $p: G \rightarrow$ $K, p(g)=(A g)$ such that $u\left(1_{K}\right)=1_{G}$ and denote the right $G$-action on $K$ by

$$
k \triangleleft g:=p((u(k) g)
$$

for $k \in$ and $g \in G$. The elements $u(k) g$ and $u(k \triangleleft g)$ differ by an element $\kappa_{k, g} \in A$ satisfying the equation

$$
u(k) g=\kappa_{k, g} u(k \triangleleft g)
$$

which furthermore satisfies the relation

$$
\kappa_{k, g_{1} g_{2}}=\kappa_{k, g_{1}} \kappa_{k \triangleleft g_{1}, g_{2}}
$$


for $k \in K$ and $g_{1}, g_{2} \in G$. Since $A$ is an abelian normal subgroup $G$, there is an induced $K$-left action on $A$ by conjugation:

$$
{ }^{k} a:=u(k) a u(k)^{-1}
$$

for $k \in K$ and $a \in A$.

Since the isomorphism class of the extension (1.1) can be classified by the cohomology class of the cocycle $F \in Z^{2}(K, A)$, i.e. a map $F: K \times K \rightarrow A$ such that

$$
\delta_{K} F\left(k_{1}, k_{2}, k_{3}\right)={ }^{k_{1}} F\left(k_{2}, k_{3}\right) F\left(k_{1} k_{2}, k_{3}\right)^{-1} F\left(k_{1}, k_{2} k_{3}\right) F\left(k_{1}, k_{2}\right)^{-1}=1,
$$

without loss of generality we will further assume that

$$
G:=A \rtimes_{F} K
$$

where the product structure of $G$ is given by the formula

$$
\left(a_{1}, k_{1}\right)\left(a_{2}, k_{2}\right):=\left(a_{1}\left({ }^{k_{1}} a_{2}\right) F\left(k_{1}, k_{2}\right), k_{1} k_{2}\right) .
$$

With this explicit choice of the group $G$, we choose the function $u: K \rightarrow G$ to be $u(k):=\left(1_{A}, k\right)$ and therefore we have that

$$
\kappa_{k_{1},\left(a, k_{2}\right)}={ }^{k_{1}} a F\left(k_{1}, k_{2}\right)
$$

thus obtaining $F\left(k_{1}, k_{2}\right)=\kappa_{k_{1},\left(1, k_{2}\right)}$. We furthermore have that for $x \in K$ and $g=(a, k) \in G$

$$
x \triangleleft g=x \triangleleft(a, k)=x k .
$$

Denote the dual group $\mathbb{A}:=\operatorname{Hom}\left(A, \mathbb{C}^{*}\right)$ and note that there is an induced $K$-right action on $\mathbb{A}$ defined as $\rho^{k}(a):=\rho\left({ }^{k} a\right)$ for $\rho \in \mathbb{A}$ and $k \in K$.

1.2. Cohomology of groups and the Lyndon-Hochschild-Serre spectral sequence. In what follows we will construct an explicit double complex whose cohomology calculates the cohomology of the group $G$, and whose associated spectral sequence recovers the Lyndon-Hochschild-Spectral (LHS) spectral sequence of the extension (1.1).

Endow the set $\operatorname{Map}\left(K, \mathbb{C}^{*}\right)$ with the left $G$-action $(g \triangleright f)(k):=f(k \triangleleft g)$ where $g \in G, k \in K$ and $f: K \rightarrow \mathbb{C}^{*}$, and consider the complex $C^{*}\left(G, \operatorname{Map}\left(K, \mathbb{C}^{*}\right)\right)$ with elements normalized chains

$C^{q}\left(G, \operatorname{Map}\left(K, \mathbb{C}^{*}\right)\right):=\left\{f: K \times G^{q} \rightarrow \mathbb{C}^{*} \mid f\left(k ; g_{1}, \ldots, g_{q}\right)=1\right.$ whenever some $\left.g_{i}=1\right\}$ and boundary map

$$
\begin{array}{r}
\left(\delta_{G} f\right)\left(k ; g_{1}, \ldots, g_{q}\right)=f\left(k \triangleleft g_{1} ; g_{2}, \ldots, g_{q}\right) \prod_{i=1}^{q-1} f\left(k ; g_{1}, . ., g_{i} g_{i+1}, \ldots, g_{q}\right)^{(-1)^{i}} \\
(1.3) \quad f\left(k ; g_{1}, \ldots, g_{q-1}\right)^{(-1)^{q}} .
\end{array}
$$

Since the natural morphism of groupoids, defined by the inclusion of the group $A$ into the action groupoid defined by the right action of $G$ on $K$, is an equivalence of categories, we have that the restriction map

$$
\psi: C^{*}\left(G, \operatorname{Map}\left(K, \mathbb{C}^{*}\right)\right) \rightarrow C^{*}\left(A, \mathbb{C}^{*}\right), \quad \psi(f)\left(a_{1}, \ldots, a_{q}\right):=f\left(1_{K} ; a_{1}, \ldots, a_{q}\right)
$$

is a morphism of complexes which induces an isomorphism in cohomology

$$
\widetilde{\psi}: H^{*}\left(G, \operatorname{Map}\left(K, \mathbb{C}^{*}\right)\right) \stackrel{\cong}{\rightarrow} H^{*}\left(A, \mathbb{C}^{*}\right) .
$$

The inverse map could be constructed at the level of cocycles as follows 
Lemma 1.1. The map $\varphi: C^{q}\left(A, \mathbb{C}^{*}\right) \rightarrow C^{q}\left(G, \operatorname{Map}\left(K, \mathbb{C}^{*}\right)\right)$

$$
\varphi(\alpha)\left(k ; g_{1}, \ldots, g_{q}\right):=\alpha\left(\kappa_{k, g_{1}}, \kappa_{k \triangleleft g_{1}, g_{2}}, \ldots, \kappa_{k \triangleleft g_{1} g_{2} \ldots g_{q-1}, g_{q}}\right)
$$

defines a map of complexes inducing an isomorphism in cohomology $\widetilde{\varphi}: H^{*}\left(A, \mathbb{C}^{*}\right) \stackrel{\cong}{\rightrightarrows}$ $H^{*}\left(G, \operatorname{Map}\left(K, \mathbb{C}^{*}\right)\right)$ which is the inverse of the map $\widetilde{\psi}$.

Proof. On the one hand we have

$$
\begin{aligned}
& \delta_{G} \varphi(\alpha)\left(k ; g_{1}, \ldots, g_{p}\right)=\varphi(\alpha)\left(k \triangleleft g_{1} ; g_{2}, \ldots, g_{q}\right) \prod_{i=1}^{q-1} \varphi(\alpha)\left(k ; g_{1}, . ., g_{i} g_{i+1}, \ldots, g_{q}\right)^{(-1)^{i}} \\
& \varphi(\alpha)\left(k ; g_{1}, \ldots, g_{q-1}\right)^{(-1)^{q}}
\end{aligned}
$$

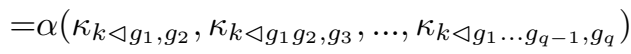

$$
\begin{aligned}
& \prod_{i=1}^{q-1} \alpha\left(\kappa_{k, g_{1}}, \kappa_{k \triangleleft g_{1}, g_{2}}, \ldots, \kappa_{k \triangleleft g_{1} \ldots g_{i-1}, g_{i} g_{i+1}}, \ldots, \kappa_{k \triangleleft g_{1} \ldots g_{q-1}, g_{q}}\right)^{(-1)^{i}} \\
& \alpha\left(\left(\kappa_{k, g_{1}}, \kappa_{k \triangleleft g_{1}, g_{2}}, \ldots, \kappa_{k \triangleleft g_{1} \ldots g_{q-2}, g_{q-1}}\right)^{(-1)^{q}}\right.
\end{aligned}
$$

and on the other

$$
\begin{aligned}
& \varphi\left(\delta_{G} \alpha\right)\left(k ; g_{1}, \ldots, g_{p}\right)=\delta_{G} \alpha\left(\kappa_{k, g_{1}}, \kappa_{k \triangleleft g_{1}, g_{2}}, \ldots, \kappa_{k \triangleleft g_{1} g_{2}, \ldots, g_{q-1}, g_{q}}\right) \\
& =\alpha\left(\kappa_{k \triangleleft g_{1}, g_{2}}, \kappa_{k \triangleleft g_{1} g_{2}, g_{3}}, \ldots, \kappa_{k \triangleleft g_{1} \ldots g_{q-1}, g_{q}}\right) \\
& \prod_{i=1}^{q-1} \alpha\left(\kappa_{k, g_{1}}, \kappa_{k \triangleleft g_{1}, g_{2}}, \ldots, \kappa_{k \triangleleft g_{1} \ldots g_{i-1}, g_{i}} \kappa_{k \triangleleft g_{1} \ldots g_{i-1} g_{i}, g_{i+1}}, \ldots, \kappa_{k \triangleleft g_{1} \ldots g_{q-1}, g_{q}}\right)^{(-1)^{i}} \\
& \alpha\left(\left(\kappa_{k, g_{1}}, \kappa_{k \triangleleft g_{1}, g_{2}}, \ldots, \kappa_{k \triangleleft g_{1} \ldots g_{q-2}, g_{q-1}}\right)^{(-1)^{q}} .\right.
\end{aligned}
$$

The equality $\delta_{G} \varphi(\alpha)=\varphi\left(\delta_{G} \alpha\right)$ follows from the identity

$$
\kappa_{k \triangleleft g_{1} \ldots g_{i-1}, g_{i} g_{i+1}}=\kappa_{k \triangleleft g_{1} \ldots g_{i-1}, g_{i}} \kappa_{k \triangleleft g_{1} \ldots g_{i-1} g_{i}, g_{i+1}} .
$$

Finally, the composition $\psi(\varphi(\alpha))=\alpha$ follows from the equation $\kappa_{1, a}=a$ for $a \in$ A.

The complex $C^{*}\left(A, \mathbb{C}^{*}\right)$ can be endowed with the structure of a right K-module by setting for $\alpha \in C^{q}\left(A, \mathbb{C}^{*}\right)$ and $k \in K$

$$
\alpha^{k}\left(a_{1}, \ldots, a_{q}\right):=\alpha\left(u(k) a_{1} u(k)^{-1}, \ldots, u(k) a_{q} u(k)^{-1}\right),
$$

and the complex $C^{*}\left(G, \operatorname{Map}\left(K, \mathbb{C}^{*}\right)\right)$ can also be endowed with the structure of a right $K$-module by setting for $f \in C^{q}\left(G, \operatorname{Map}\left(K, \mathbb{C}^{*}\right)\right)$ and $k \in K$

$$
(f \triangleleft k)\left(x ; g_{1}, \ldots, g_{q}\right):=f\left(k x ; g_{1}, \ldots, g_{q}\right) .
$$

The map $\varphi$ fails to be a $K$-module map; nevertheless it induces a $K$-module map at the level of cohomology

Lemma 1.2. The isomorphism $\widetilde{\varphi}: H^{*}\left(A, \mathbb{C}^{*}\right) \stackrel{\cong}{\rightarrow} H^{*}\left(G, \operatorname{Map}\left(K, \mathbb{C}^{*}\right)\right)$ is an isomorphism of $K$-modules.

Proof. Take $\alpha \in Z^{q}\left(A, \mathbb{C}^{*}\right)$ and $k \in K$. We claim that $\psi(\varphi(\alpha) \triangleleft k)=\alpha^{k}$, and since $\psi\left(\varphi\left(\alpha^{k}\right)\right)=\alpha^{k}$, we conclude that $\varphi(\alpha) \triangleleft k$ and $\varphi\left(\alpha^{k}\right)$ are cohomologous. Now, let 
us calculate

$$
\begin{aligned}
\psi(\varphi(\alpha) \triangleleft k)\left(a_{1}, \ldots, a_{q}\right) & =(\varphi(\alpha) \triangleleft k)\left(1 ; a_{1}, \ldots, a_{q}\right) \\
& =\varphi(\alpha)\left(k ; a_{1}, \ldots, a_{q}\right) \\
& =\alpha\left(\kappa_{k, a_{1}}, \kappa_{k \triangleleft a_{1}, a_{2}}, \ldots, \kappa_{k \triangleleft a_{1} a_{2}, \ldots, a_{q-1}, a_{q}}\right) \\
& =\alpha\left(\kappa_{k, a_{1}}, \kappa_{k, a_{2}}, \ldots, \kappa_{k, a_{q}}\right) \\
& =\alpha\left(u(k) a_{1} u(k)^{-1}, u(k) a_{2} u(k)^{-1}, \ldots, u(k) a_{q} u(k)^{-1}\right) \\
& =\alpha^{k}\left(a_{1}, a_{2}, \ldots, a_{q}\right) ;
\end{aligned}
$$

the lemma follows.

1.2.1. Double complex. Since $C^{*}\left(G, \operatorname{Map}\left(K, \mathbb{C}^{*}\right)\right)$ is a complex of right $K$-modules, we can consider the complexes

$$
C^{*}\left(K, C^{q}\left(G, \operatorname{Map}\left(K, \mathbb{C}^{*}\right)\right)\right)
$$

with $C^{p}\left(K, C^{q}\left(G, \operatorname{Map}\left(K, \mathbb{C}^{*}\right)\right)\right.$ consisting of normalized cochains

$$
\left\{f: K^{p} \rightarrow C^{q}\left(G, \operatorname{Map}\left(K, \mathbb{C}^{*}\right)\right) \mid f\left(k_{1}, \ldots, k_{p}\right)=1 \text { whenever some } k_{i}=1\right\}
$$

and whose differentials

$\left(\delta_{K} f\right)\left(k_{1}, \ldots, k_{p}\right)=f\left(k_{2}, \ldots, k_{p}\right) \prod_{i=1}^{p-1} f\left(k_{1}, \ldots, k_{i} k_{i+1}, \ldots, k_{p}\right)^{(-1)^{i}}\left(f\left(k_{1}, \ldots, k_{p-1}\right) \triangleleft k_{p}\right)^{(-1)^{p}}$.

These complexes assemble into a double complex $C^{p, q}:=C^{p}\left(K, C^{q}\left(G, \operatorname{Map}\left(K, \mathbb{C}^{*}\right)\right)\right)$. Let us denote by $\operatorname{Tot}\left(C^{*, *}\right)$ the total complex associated to the double complex and let $\delta_{T o t}:=\delta_{K} \oplus\left(\delta_{G}\right)^{(-1)^{p}}$ be its differential.

We may filter the total complex by the degree of the $G$ cochains, thus obtaining a spectral sequence whose first page becomes

$$
E_{1}^{p, q}=H^{p}\left(K, C^{q}\left(G, \operatorname{Map}\left(K, \mathbb{C}^{*}\right)\right)\right) .
$$

Since the $K$-modules $C^{q}\left(G, \operatorname{Map}\left(K, \mathbb{C}^{*}\right)\right)$ are free $K$-modules, we conclude that the first page localizes on the $y$-axis,

$$
E_{1}^{0, q}=H^{0}\left(K, C^{q}\left(G, \operatorname{Map}\left(K, \mathbb{C}^{*}\right)\right)\right)=C^{q}\left(G, \operatorname{Map}\left(K, \mathbb{C}^{*}\right)\right)^{K} \cong C^{q}\left(G, \mathbb{C}^{*}\right)
$$

and $E_{1}^{p, q}=0$ for $p>0$. The spectral sequence collapses at the second page, with the only surviving elements on the $y$-axis

$$
E_{2}^{0, q}=H^{q}\left(G, \mathbb{C}^{*}\right)
$$

Hence we have

Proposition 1.3. The inclusion of $K$-invariant cochains

$$
C^{*}\left(G, \operatorname{Map}\left(K, \mathbb{C}^{*}\right)\right)^{K} \hookrightarrow \operatorname{Tot}\left(C^{*}\left(K, C^{*}\left(G, \operatorname{Map}\left(K, \mathbb{C}^{*}\right)\right)\right)\right)
$$

is a quasi-isomorphism. Therefore the cohomology groups

$$
H^{*}\left(G, \mathbb{C}^{*}\right) \stackrel{\cong}{\rightrightarrows} H^{*}\left(\operatorname{Tot}\left(C^{*}\left(K, C^{*}\left(G, \operatorname{Map}\left(K, \mathbb{C}^{*}\right)\right)\right)\right)\right.
$$

are canonically isomorphic. 
Filtering the double complex by the degree of the $K$ cochains we obtain the Lyndon-Hochschild-Serre spectral sequence associated to the group extension $1 \rightarrow$ $A \rightarrow G \rightarrow K \rightarrow 1$ (see [7, $\S 7.2]$ and references therein). The first page becomes

$$
E_{1}^{p, q}=C^{p}\left(K, H^{q}\left(G, \operatorname{Map}\left(K, \mathbb{C}^{*}\right)\right)\right)
$$

and the second page becomes

$$
E_{2}^{p, q}=H^{p}\left(K, H^{q}\left(G, \operatorname{Map}\left(K, \mathbb{C}^{*}\right)\right)\right)
$$

Since the projection map $\widetilde{\psi}: H^{q}\left(G, \operatorname{Map}\left(K, \mathbb{C}^{*}\right)\right) \stackrel{\cong}{\rightarrow} H^{q}\left(A, \mathbb{C}^{*}\right)$ is an isomorphism of $K$-modules, we conclude

Proposition 1.4 (LHS spectral sequence). Filtering the total complex by the degree of the $K$-chains, we obtain a spectral sequence whose second page is

$$
E_{2}^{p, q} \cong H^{p}\left(K, H^{q}\left(A, \mathbb{C}^{*}\right)\right)
$$

and that converges to $H^{*}\left(G, \mathbb{C}^{*}\right)$.

We will denote $d_{i}: E_{i}^{p, q} \rightarrow E_{i}^{p+i, q-i+1}$ the differentials of this spectral sequence.

1.3. Tensor categories. Following [2, §1], a tensor category consist of $\left(\mathcal{C}, \otimes, 1_{\mathcal{C}}, \alpha, \lambda, \rho\right)$ where $\mathcal{C}$ is a category, $\otimes: \mathcal{C} \times \mathcal{C} \rightarrow \mathcal{C}$ is a bifunctor, $\alpha$ is the associativity constraint i.e. a functorial isomorphism $\alpha_{U V W}:(U \otimes V) \otimes W \stackrel{\sim}{\rightarrow} U \otimes(V \otimes W)$ of functors $\mathcal{C} \times \mathcal{C} \times \mathcal{C} \rightarrow \mathcal{C}, 1_{\mathcal{C}} \in \mathrm{Ob}(\mathcal{C})$ is a unit element and $\lambda, \rho$ are functorial isomorphisms $\lambda_{V}: 1_{\mathcal{C}} \otimes V \stackrel{\sim}{\rightarrow} V, \rho_{V}: V \otimes 1_{\mathcal{C}} \stackrel{\sim}{\rightarrow} V$, satisfying the pentagon axiom

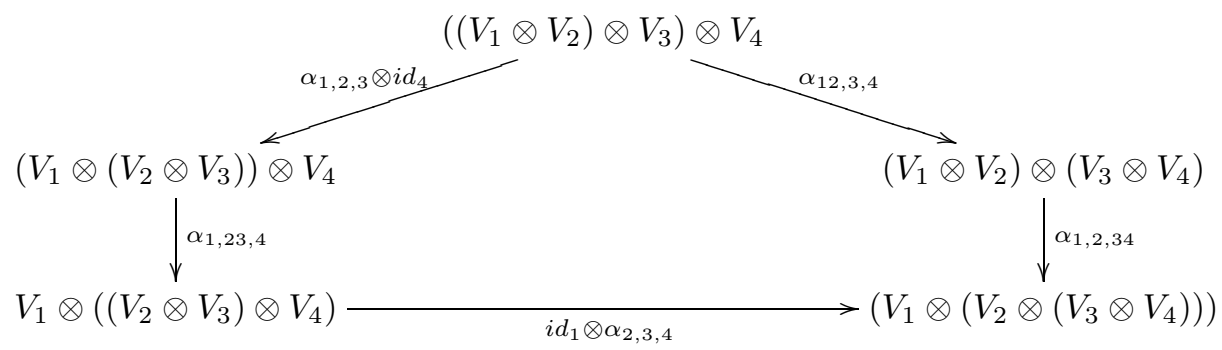

and the triangle axiom

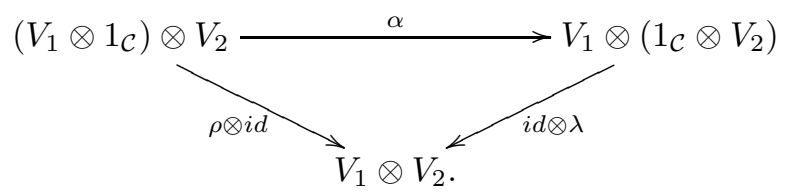

1.4. The fusion category $\operatorname{Vect}(G, \omega)$. A fusion category over $\mathbb{C}$ is a rigid semisimple $\mathbb{C}$-linear tensor category, with only finitely many isomorphism classes of simple objects, such that the endomorphisms of the unit object is $\mathbb{C}$ (see [5]).

For $G$ a finite group and a 3 -cocycle $\omega \in Z^{3}\left(G, \mathbb{C}^{*}\right)$, define the category $\operatorname{Vect}(G, \omega)$ by setting that its objects are $G$-graded complex vector spaces $V=\bigoplus_{g \in G} V_{g}$, whose tensor product is

$$
(V \otimes W)_{g}:=\bigoplus_{h k=g} V_{h} \otimes W_{k}
$$

whose associativity constraint is $\alpha_{V_{g}, V_{h}, V_{k}}=\omega(g, h, k) \gamma$ with $\gamma((x \otimes y) \otimes z)=$ $x \otimes(y \otimes z)$, and whose left and right unit isomorphisms are $\lambda_{V_{g}}=\omega(1,1, g)^{-1} i d_{V_{g}}$ 
and $\rho_{V_{g}}=\omega(g, 1,1) i d_{V_{g}}$. The category $\operatorname{Vect}(G, \omega)$ is a fusion category where the simple objects are the 1-dimensional vector spaces.

We will assume that all group cochains are normalized, and hence the left and right unit isomorphisms become identities.

For convenience we will work with a category $\mathcal{V}(G, \omega)$ which is skeletal, i.e. one on which isomorphic objects are equal, and which is equivalent to $\operatorname{Vect}(G, \omega)$. The category $\mathcal{V}(G, \omega)$ has for simple objects the elements $g$ of the group $G$, the tensor product is $g \otimes h=g h$ and the associativity isomorphisms are $\omega(g, h, k) i d_{g h k}$.

A finite tensor category is called pointed if all its simple objects are invertible. It is thus easy to see that any finite tensor category which is pointed is equivalent to $\operatorname{Vect}(G, \omega)$ for some finite group $G$ and some 3-cocycle $\omega$.

1.5. Module Categories. Following [12, §2.3], a right module category over the tensor category $\left(\mathcal{C}, \otimes, 1_{\mathcal{C}}, \alpha, \lambda, \rho\right)$ consists of $(\mathcal{M}, \otimes, \mu, \tau)$ where $\mathcal{M}$ is a category, $\otimes: \mathcal{M} \times \mathcal{C} \rightarrow \mathcal{M}$ is an exact bifunctor, $\mu_{M, X, Y}: M \otimes(X \otimes Y) \stackrel{\sim}{\rightarrow}(M \otimes X) \otimes Y$ is a functorial associativity and $\tau_{M}: M \otimes 1_{\mathcal{C}} \stackrel{\sim}{\rightarrow} M$ is a unit isomorphism for any $X, Y \in \mathcal{C}, M \in \mathcal{M}$, satisfying the pentagon axiom

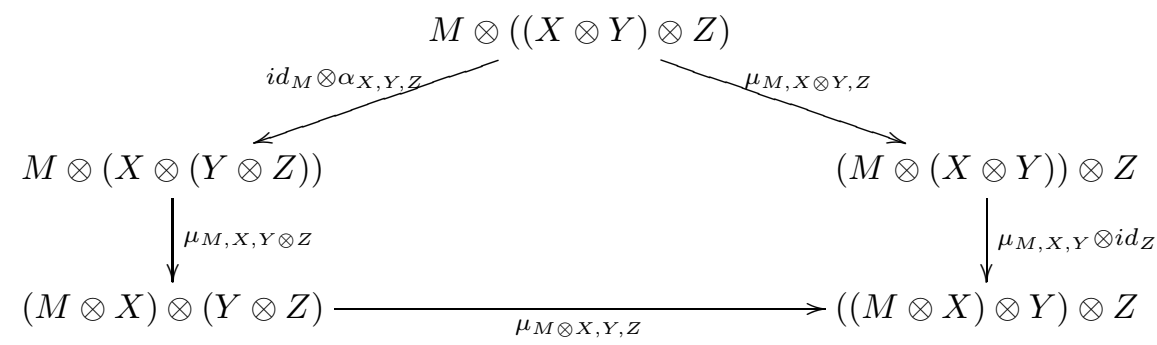

and the triangle axiom

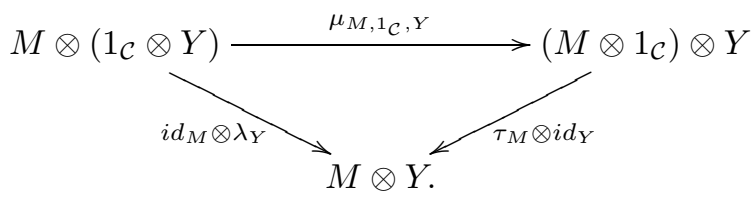

A module functor $(F, \gamma):\left(\mathcal{M}_{1}, \mu^{1}, \tau^{1}\right) \rightarrow\left(\mathcal{M}_{2}, \mu^{2}, \tau^{2}\right)$ between two module categories consist of a functor $F: \mathcal{M}_{1} \rightarrow \mathcal{M}_{2}$ and a functorial isomorphism $\gamma_{M, X}$ : $F(M \otimes X) \rightarrow F(M) \otimes X$ for any $X \in \mathcal{C}, M \in \mathcal{M}$, satisfying the pentagon axiom

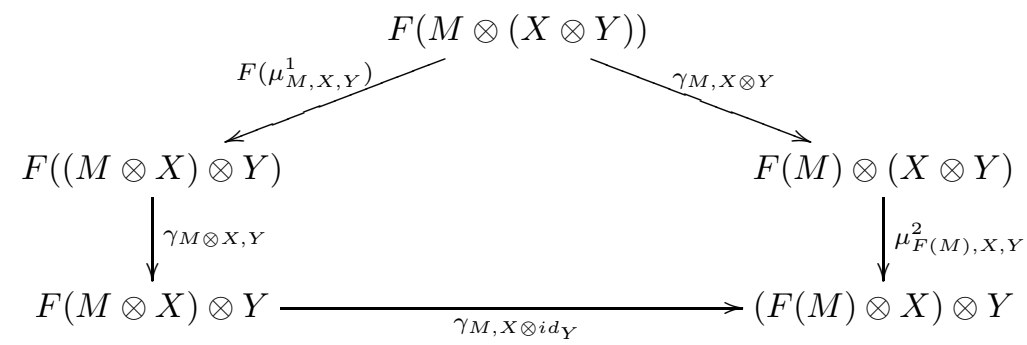


and the triangle axiom

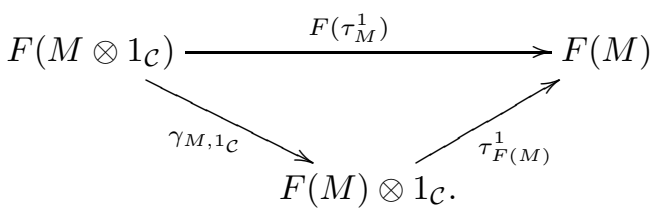

Two module categories $\mathcal{M}_{1}$ and $\mathcal{M}_{2}$ over $\mathcal{C}$ are equivalent if there exist a module functor between the two which is moreover an equivalence of categories. The direct sum $\mathcal{M}_{1} \oplus \mathcal{M}_{2}$ is the module category with the obvious structure. A module category is indecomposable if it is not equivalent to the direct sum of two nontrivial module categories.

A natural module transformation $\eta:\left(F^{1}, \gamma^{1}\right) \rightarrow\left(F^{2}, \gamma^{2}\right)$ consist of a natural transformation $\eta: F^{1} \rightarrow F^{2}$ such that the square

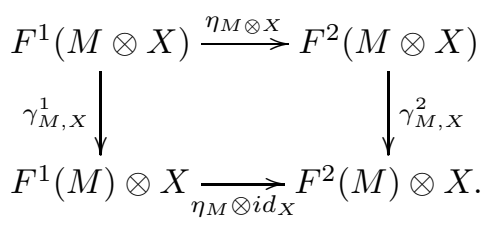

commutes for all $M \in \mathcal{M}$ and $X \in \mathcal{C}$.

1.6. Indecomposable module categories over $\mathcal{V}(G, \omega)$. Let $\mathcal{M}$ be a skeletal right module category over $\mathcal{V}(G, \omega)$. The set of simple objects of $\mathcal{M}$ is a transitive right $G$-set and therefore it can be identified with the coset $K:=A \backslash G$ for $A$ a subgroup of $G$. The isomorphisms $\mu_{k, g_{1}, g_{2}}$ for $k \in K$ and $g_{1}, g_{2} \in G$ are scalars, and we can assemble these scalars as an element

$$
\mu \in C^{2}\left(G, \operatorname{Map}\left(K, \mathbb{C}^{*}\right)\right), \quad \mu\left(k ; g_{1}, g_{2}\right):=\mu_{k, g_{1}, g_{2}} .
$$

The pentagon axiom (1.4) translates into the equation

$$
\omega\left(g_{1}, g_{2}, g_{3}\right) \mu\left(k ; g_{1}, g_{2} g_{3}\right) \mu\left(k \triangleleft g_{1} ; g_{2}, g_{3}\right)=\mu\left(k ; g_{1} g_{2}, g_{3}\right) \mu\left(k ; g_{1}, g_{2}\right),
$$

which in view of the definition of the differential $\delta_{G}$ in (1.3) becomes

$$
\delta_{G} \mu^{-1}=\pi^{*} \omega
$$

where $\pi^{*} \omega \in C^{3}\left(G, \operatorname{Map}\left(K, \mathbb{C}^{*}\right)\right)^{K}$ is the $K$-invariant cocycle defined by $\omega$, i.e.

$$
\pi^{*} \omega\left(k ; g_{1}, g_{2}, g_{3}\right):=\omega\left(g_{1}, g_{2}, g_{3}\right) .
$$

Since $\mu$ is normalized and the unit constraint in $\mathcal{V}(G, \omega)$ is trivial, we have that the triangle axiom (1.5) implies that the unit constraint in $\mathcal{M}$ is trivial.

Denote this skeletal module category $\mathcal{M}=\mathcal{M}(A \backslash G, \mu)$. Note that two $\mathcal{V}(G, \omega)$ module categories $\mathcal{M}_{1}=\mathcal{M}\left(A_{1} \backslash G, \mu_{1}\right)$ and $\mathcal{M}_{2}=\mathcal{M}\left(A_{2} \backslash G, \mu_{2}\right)$ are equivalent if and only if there exist a right $G$-equivariant isomorphism $F: A_{1} \backslash G \stackrel{\cong}{\rightrightarrows} A_{2} \backslash G$ and an element $\gamma \in C^{1}\left(G, \operatorname{Map}\left(A_{1} \backslash G, \mathbb{C}^{*}\right)\right)$ such that

$$
\gamma\left(A_{1} g ; g_{1} g_{2}\right) \mu_{2}\left(F\left(A_{1} g\right) ; g_{1}, g_{2}\right)=\mu_{1}\left(A_{1} g ; g_{1}, g_{2}\right) \gamma\left(A_{1} g g_{1} ; g_{2}\right) \gamma\left(A_{1} g ; g_{1}\right) \text {. }
$$

This information implies that $A_{1}$ and $A_{2}$ are conjugate subgroups of $G$ and that

$$
\delta_{G} \gamma=\frac{F^{*} \mu_{2}}{\mu_{1}} .
$$


In the case that $A=A_{1}=A_{2}$, the $G$-equivariant isomorphisms are parameterized by the elements of the group $A \backslash N_{G}(A)$, and the equation $\delta_{G} \gamma=\frac{F^{*} \mu_{2}}{\mu_{1}}$ implies that $\frac{F^{*} \mu_{2}}{\mu_{1}}$ is trivial in $H^{2}\left(G, \operatorname{Map}\left(A \backslash G, \mathbb{C}^{*}\right)\right)$. Since we know that $\widetilde{\psi}$ : $H^{2}\left(G, \operatorname{Map}\left(A \backslash G, \mathbb{C}^{*}\right)\right) \stackrel{\cong}{\rightrightarrows} H^{2}\left(A, \mathbb{C}^{*}\right)$ is an isomorphism, we can conclude that the isomorphism classes of module categories over $\mathcal{V}(G, \omega)$ may be parameterized (in a non-canonical manner) by pairs $([A],[\psi(\mu)])$ where $[A]$ is a conjugacy class of subgroups of $G$, and $[\psi(\mu)]$ is a representative of a cohomology class in the group of invariants $H^{2}\left(A, \mathbb{C}^{*}\right) / N_{G}(A)$.

1.7. Dual category. Let $\mathcal{C}$ be a tensor category and $\mathcal{M}$ an indecomposable right module category. The dual category $\mathcal{C}_{\mathcal{M}}^{*}:=\operatorname{Fun}_{\mathcal{C}}(\mathcal{M}, \mathcal{M})$ is the category whose objects are module functors from $\mathcal{M}$ to itself and whose morphisms are natural module transformations.

The category $\mathcal{C}_{\mathcal{M}}^{*}$ becomes a tensor category by composition of functors, namely for $\left(\gamma^{1}, F_{1}\right),\left(\gamma^{2}, F_{2}\right) \in \operatorname{Obj}\left(\mathcal{C}_{\mathcal{M}}^{*}\right)$ where $\gamma^{1}, \gamma^{2}$ represent the module structures on the functors $F_{1}$ and $F_{2}$ respectively, we define the tensor structure by $\left(\gamma^{1}, F_{1}\right) \otimes$ $\left(\gamma^{2}, F_{2}\right):=\left(\gamma, F_{1} \circ F_{2}\right)$ where the module structure $\gamma$ is defined by $\gamma_{M, X}:=$ $\gamma_{F_{2}(M), X}^{1} \circ F_{1}\left(\gamma_{M, X}^{2}\right)$ for $M \in \mathcal{M}$ and $X \in \mathcal{C}$. For two morphisms $\eta:\left(\gamma^{1}, F_{1}\right) \rightarrow$ $\left(\gamma^{2}, F_{2}\right)$ and $\eta^{\prime}:\left(\gamma^{\prime 1}, F_{1}^{\prime}\right) \rightarrow\left(\gamma^{\prime 2}, F_{2}^{\prime}\right)$ in $\mathcal{C}_{\mathcal{M}}^{*}$ their tensor product is $\left(\eta \otimes \eta^{\prime}\right)(M):=$ $\eta_{F_{2}^{\prime}(M)} \circ F_{1}\left(\eta_{M}^{\prime}\right)$.

Whenever $\mathcal{C}$ and $\mathcal{M}$ are semisimple, the dual category $\mathcal{C}_{\mathcal{M}}^{*}$ is semisimple 11, $\S 2.2$. Moreover, since $\mathcal{M}$ is itself a left module category over $\mathcal{C}_{\mathcal{M}}^{*}$ it has been shown in [12, Cor. 4.1] that the double dual is tensor equivalent to the original category, i.e. $\left(\mathcal{C}_{\mathcal{M}}^{*}\right)_{\mathcal{M}}^{*} \simeq \mathcal{C}$. Furthermore, the module categories of $\mathcal{C}$ and of $\mathcal{C}_{\mathcal{M}}^{*}$ are in canonical bijection [11, Prop. 2.1] by the following maps. For $\mathcal{M}_{1}$ a module category over $\mathcal{C}$, the category $\operatorname{Fun}_{\mathcal{C}}\left(\mathcal{M}_{1}, \mathcal{M}\right)$ of module functors from $\mathcal{M}_{1}$ to $\mathcal{M}$ is a left module category of $\mathcal{C}_{\mathcal{M}}^{*}=\operatorname{Fun}_{\mathcal{C}}(\mathcal{M}, \mathcal{M})$ via the composition of functors. Conversely, if $\mathcal{M}_{2}$ is a left module category over $\mathcal{C}_{\mathcal{M}}^{*}$, then $\operatorname{Fun}_{\mathcal{C}_{\mathcal{M}}^{*}}\left(\mathcal{M}, \mathcal{M}_{2}\right)$ is a right module category over $\operatorname{Fun}_{\mathcal{C}_{\mathcal{M}}^{*}}(\mathcal{M}, \mathcal{M})=\left(\mathcal{C}_{\mathcal{M}}^{*}\right)_{\mathcal{M}}^{*} \simeq \mathcal{C}$ via composition of functors. These maps are inverse from each other.

1.8. Center of a tensor category. The center $\mathcal{Z}(\mathcal{C})$ of the tensor category $\mathcal{C}$ is the category whose objects are pairs $(X, \eta)$ where $X$ is an object in $\mathcal{C}$ and $\eta$ is a functorial set of isomorphisms $\eta_{Y}: X \otimes Y \rightarrow Y \otimes X$ such that the hexagon diagram

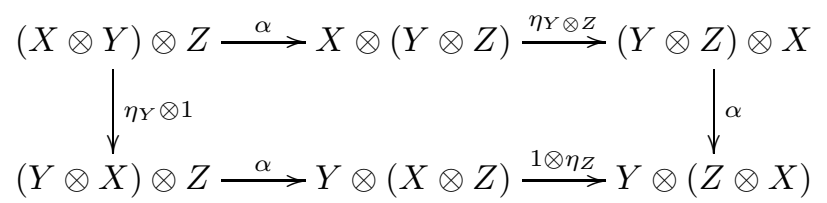

and the triangle diagram

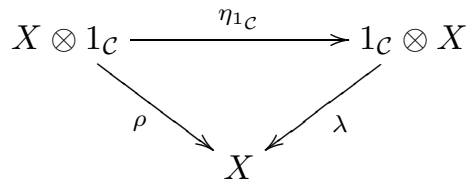


are commutative. A morphism $f:(X, \eta) \rightarrow(Y, \nu)$ consists of a morphism $f: X \rightarrow$ $Y$ for which the diagram

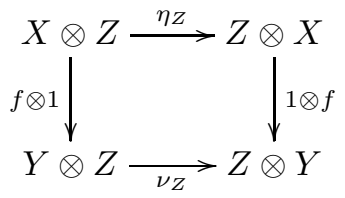

commutes for any object $Z$ in $\mathcal{C}$. The tensor structure is defined as $(X, \eta) \otimes(Y, \nu):=$ $(X \otimes Y, \gamma)$ where $\gamma_{Z}$ is defined as the composition

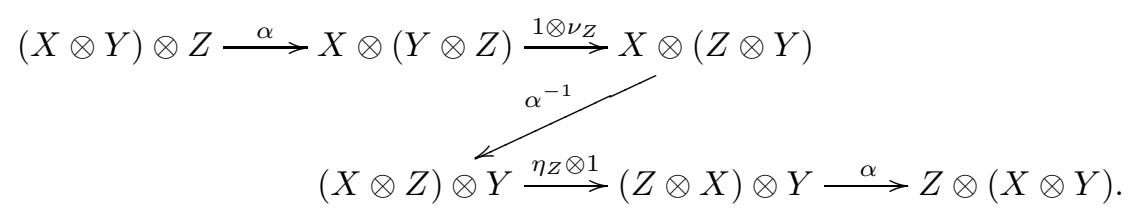

The center $\mathcal{Z}(\mathcal{C})$ is moreover braided and the braiding for the pair $(X, \eta),(Y, \nu)$ is precisely the map $\eta_{Y}$.

The center $\mathcal{Z}(\operatorname{Vect}(G, \omega))$ of the tensor category $\operatorname{Vect}(G, \omega)$ contains the information necessary for constructing the quasi-Hopf algebra that is known as the Twisted Drinfeld Double $D^{\omega}(G)$ of the group $G$ twisted by $\omega$ (see $[4, \S 3.2]$ ).

1.9. Weak Morita equivalence of tensor categories. Two tensor categories $\mathcal{C}$ and $\mathcal{D}$ are weakly Morita equivalent if there exists an indecomposable right module category $\mathcal{M}$ over $\mathcal{C}$ such that $\mathcal{C}_{\mathcal{M}}^{*}$ and $\mathcal{D}$ are tensor equivalent [9, Def 4.2]. In [9, Prop. 4.6] it is shown that weak Morita equivalence is an equivalence relation, and in [6, Thm. 3.1] it is shown that two tensor categories are weak Morita equivalent if and only if their centers are braided equivalent. In particular we have that for $\mathcal{M}$ an indecomposable module category over $\mathcal{C}$ there is a canonical equivalence of braided tensor categories $\mathcal{Z}(\mathcal{C}) \simeq \mathcal{Z}\left(\mathcal{C}_{\mathcal{M}}^{*}\right)[11$, Prop. 2.2].

\section{The dual of $\mathcal{V}(G, \omega)$ With Respect to $\mathcal{M}(A \backslash G, \mu)$}

Let us consider the tensor category $\mathcal{C}=\mathcal{V}(G, \omega)$ and the right module category $\mathcal{M}=\mathcal{M}(A \backslash G, \mu)$ described in $\$ 1.6$. In this chapter we will review the main results of [10] where explicit conditions are stated under which the dual category $\mathcal{C}_{\mathcal{M}}^{*}$ is pointed. For the sake of completeness and clarity we will review the constructions done in $\S 3$ and $\S 4$ of [10] and we will reinterpret the equations given there in the terminology that we have set up in $\$ 1.1$ and $\$ 1.2$.

2.1. Conditions for $\mathcal{C}_{\mathcal{M}}^{*}$ to be pointed. Let us setup some notation for this section: let $K:=A \backslash G, u: K \rightarrow G$ satisfy $p \circ u=1_{G}$ and $u\left(p\left(1_{G}\right)\right)=1_{G}$ for $p: G \rightarrow K$ the projection, $\kappa: K \times G \rightarrow A$ satisfy $u(k) g=\kappa_{k, q} u(k \triangleleft g)$ and $K^{A}$ the elements of $K$ fixed under the conjugation by elements of $A$. The module category $\mathcal{M}(A \backslash G, \mu)$ is the skeletal category whose simple objects are the elements of $K=A \backslash G$, whose tensor structure is $k \otimes g:=k \triangleleft g$ for $k \in K$ and $g \in G$ and whose associativity constraint $\mu$ satisfies $\delta_{G} \mu^{-1}=\pi^{*} \omega$, see (1.6) In what follows we will focus on parametrizing the invertible objects of $\mathcal{C}_{\mathcal{M}}^{*}$.

Following [10, Lemma 3.2] any invertible module functor in $\mathcal{C}_{\mathcal{M}}^{*}$ is of the form $\left(F_{y}, \gamma\right)$ where the functor $F_{y}: \mathcal{M} \rightarrow \mathcal{M}$ is the one that extends the $G$-equivariant 
map $f_{y}: K \rightarrow K, f_{y}(k)=p(u(y) u(k))$ for $y \in K^{A}$, and $\gamma$ is a functorial isomorphism $\gamma_{k, g}: F_{y}(k \otimes g) \cong F_{y}(k) \otimes g$ that satisfies the pentagon axiom. Writing $\gamma_{k, g}:=\gamma(k ; g) i d_{p(u(y) u(k \triangleleft g))}$ for $\gamma \in C^{1}\left(G, \operatorname{Map}\left(K, \mathbb{C}^{*}\right)\right)$ we have that the pentagon axiom of a module functor translates into the equation

$$
\mu\left(k ; g_{1}, g_{2}\right) \gamma\left(k \triangleleft g_{1} ; g_{2}\right) \gamma\left(k ; g_{1}\right)=\gamma\left(k ; g_{1} g_{2}\right) \mu\left(f_{y}(k) ; g_{1}, g_{2}\right)
$$

which can also be written as

$$
\delta_{G} \gamma\left(k ; \gamma_{1}, \gamma_{2}\right)=\frac{\mu\left(f_{y}(k) ; g_{1}, g_{2}\right)}{\mu\left(k ; g_{1}, g_{2}\right)} .
$$

The inverse of $\left(F_{y}, \gamma\right)$ is the module functor $\left(F_{p\left(u(y)^{-1}\right)}, \bar{\gamma}\right)$ with

$$
\bar{\gamma}(k ; g):=\gamma\left(p\left(u(y)^{-1} u(x)\right)^{-1} ; g\right)^{-1} .
$$

Defining for each $y \in K^{A}$ the set

$$
\text { Fun }_{y}:=\left\{\gamma \in C^{1}\left(G, \operatorname{Map}\left(K, \mathbb{C}^{*}\right)\right) \mid \delta_{G} \gamma\left(k ; g_{1}, g_{2}\right)=\frac{\mu\left(f_{y}(k) ; g_{1}, g_{2}\right)}{\mu\left(k ; g_{1}, g_{2}\right)}\right\}
$$

for all $k \in K$ and $g_{1}, g_{2} \in G$, we have that the set of invertible objects of $\mathcal{C}_{\mathcal{M}}^{*}$ are precisely the module functors $\left(F_{y}, \gamma\right)$ where $y \in K^{A}$ and $\gamma \in F u n_{y}$. To simplify the notation we will denote such module functor by the pair $(y, \gamma)$.

Two invertible module functors $\left(y_{1}, \gamma^{1}\right)$ and $\left(y_{2}, \gamma^{2}\right)$ in $\mathcal{C}_{\mathcal{M}}^{*}$ are isomorphic if and only if $y_{1}=y_{2}$ and if there exists natural transformation parameterized by a map $\eta \in C^{0}\left(G, \operatorname{Map}\left(K, \mathbb{C}^{*}\right)\right)$ satisfying the equation

$$
\gamma^{1}(k ; g) \eta(k)=\eta(k \triangleleft g) \gamma^{2}(k ; g)
$$

for all $k \in K$ and $g \in G$. These equations can be rewritten as the equation

$$
\delta_{G} \eta=\frac{\gamma^{2}}{\gamma^{1}}
$$

in $C^{1}\left(G, \operatorname{Map}\left(K, \mathbb{C}^{*}\right)\right)$. Therefore for each $y \in K^{A}$ we may define an equivalence

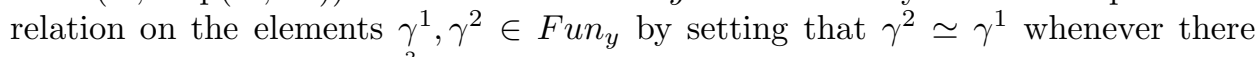
exist $\eta$ such that $\delta_{G} \eta=\frac{\gamma^{2}}{\gamma^{1}}$; denote the by $\overline{F u n}_{y}$ the associated set of equivalence classes.

For each $y \in K^{A}$ let us choose an element $\gamma_{y} \in F u n_{y}$, and note that the maps

$$
\operatorname{Fun}_{y} \rightarrow Z^{1}\left(G, \operatorname{Map}\left(K, \mathbb{C}^{*}\right)\right), \beta \mapsto \frac{\beta}{\gamma_{y}}, \quad Z^{1}\left(G, \operatorname{Map}\left(k, \mathbb{C}^{*}\right)\right) \rightarrow F u n_{y}, \epsilon \mapsto \epsilon \gamma_{y}
$$

are inverse to each other. Therefore we obtain bijections

$$
\overline{\text { Fun }}_{y} \cong H^{1}\left(G, \operatorname{Map}\left(K, \mathbb{C}^{*}\right)\right) \cong H^{1}\left(A, \mathbb{C}^{*}\right)=\mathbb{A}
$$

which are realized by the maps

$$
\begin{array}{ll}
\zeta_{y}: \mathbb{A} \rightarrow \text { Fun }_{y}, & \zeta_{y}(\rho):=\gamma_{y} \varphi(\rho) \\
\theta_{y}: \text { Fun }_{y} \rightarrow \mathbb{A}, & \theta_{y}(\beta):=\psi\left(\beta / \gamma_{y}\right) .
\end{array}
$$

Recall from [5, Def. 2.2] that the global dimension $\operatorname{dim}(\mathcal{C})$ of a fusion category $\mathcal{C}$ is the sum of the squared norms of its simple objects, and note that by $[5$, Thm. 2.15 ] we have $\operatorname{dim}\left(\mathcal{C}_{\mathcal{M}}^{*}\right)=\operatorname{dim}(\mathcal{C})$ whenever $\mathcal{C}$ is a fusion category and $\mathcal{M}$ is an indecomposable module category over $\mathcal{C}$. 
Let us suppose now that the dual category $\mathcal{C}_{\mathcal{M}}^{*}=\mathcal{V}(G, \omega)_{\mathcal{M}(A \backslash G, \mu)}^{*}$ is pointed. Therefore its global dimension

$$
\operatorname{dim}\left(\mathcal{C}_{\mathcal{M}}^{*}\right)=|\mathbb{A}|\left|K^{A}\right|
$$

must be equal to the number of isomorphic classes of invertible objects, since on pointed categories all simple objects are invertible. On the other hand, by 5 , Thm. 2.15 ] we have that $\operatorname{dim}\left(\mathcal{C}_{\mathcal{M}}^{*}\right)=\operatorname{dim}(\mathcal{C})$ and $\operatorname{dim}(\mathcal{C})=|G|$. Therefore in order for the category $\mathcal{C}_{\mathcal{M}}^{*}$ to be pointed it is necessary that $|\mathbb{A}|\left|K^{A}\right|=|G|$. Since $|G|=|A||K|$, $|\mathbb{A}| \leq|A|$ and $\left|K^{A}\right| \leq|K|$, the equality holds if and only if $A$ is abelian, thus having that $|\mathbb{A}|=|A|$, and if $A$ is normal in $G$ and $K^{A}=K$.

On the other hand, if $A$ is abelian and normal on $G$, then the number of isomorphism classes of invertible objects in $\mathcal{C}_{\mathcal{M}}^{*}$ is $|\mathbb{A}||K|=|G|$. Since $\operatorname{dim}\left(\mathcal{C}_{\mathcal{M}}^{*}\right)=$ $\operatorname{dim}(\mathcal{C})=|G|$ then we have that the set of isomorphism classes of invertible objects exhaust the set of simple elements, and therefore $\mathcal{C}_{\mathcal{M}}^{*}$ must be pointed.

Summarizing we have

Theorem 2.1. [10, Thm 3.4] The tensor category $\mathcal{C}_{\mathcal{M}}^{*}=\mathcal{V}(G, \omega)_{\mathcal{M}(A \backslash G, \mu)}^{*}$ is pointed if and only if $A$ is abelian and normal in $G$ and the cohomology class $\left[\frac{\mu \triangleleft y}{\mu}\right]$ is trivial in $H^{2}\left(G, \operatorname{Map}\left(K, \mathbb{C}^{*}\right)\right)$ for all $y \in K$.

Note that since $A$ is normal in $G$, we may use the notation introduced in $\$ 1.2$ so that $\mu\left(f_{y}(k) ; g_{1}, g_{2}\right)=\mu\left(y k ; g_{1}, g_{2}\right)=(\mu \triangleleft y)\left(k ; g_{1}, g_{2}\right)$. Since we have that $\delta_{G} \mu^{-1}=$ $\pi^{*} \omega=\delta_{G}\left(\mu^{-1} \triangleleft y\right)$, the quotient $\frac{\mu \triangleleft y}{\mu}$ defines a cocycle in $Z^{2}\left(G, \operatorname{Map}\left(K, \mathbb{C}^{*}\right)\right)$. The equation $\delta_{G} \gamma_{y}=\frac{\mu \triangleleft y}{\mu}$ implies that the quotient is trivial in cohomology.

2.2. The Grothendieck ring of the pointed category $\mathcal{C}_{\mathcal{M}}^{*}$. From now on we will assume that the dual category $\mathcal{C}_{\mathcal{M}}^{*}$ is pointed. Therefore we have that $A$ is abelian and normal in $G$ and that we can choose elements $\gamma_{y} \in C^{1}\left(G, \operatorname{Map}\left(K, \mathbb{C}^{*}\right)\right)$ for each $y \in K$ such that $\delta_{G} \gamma_{y}=\frac{\mu \triangleleft y}{\mu}$.

The Grothendieck ring $K_{0}\left(\mathcal{C}_{\mathcal{M}}^{*}\right)$ of the category $\mathcal{C}_{\mathcal{M}}^{*}$ is the ring defined by the semi-ring whose elements are the isomorphism classes of objects and whose product is the one induced by the tensor product. Since $\mathcal{C}_{\mathcal{M}}^{*}$ is pointed then $K_{0}\left(\mathcal{C}_{\mathcal{M}}^{*}\right)$ is isomorphic to the group ring $\mathbb{Z}[\Lambda]$ for some finite group $\Lambda$. In this section we will recall the construction of this isomorphism carried out in [10, Thm. 4.5].

The tensor product of two invertible elements $\left(y_{1}, \gamma^{1}\right),\left(y_{2}, \gamma^{2}\right)$ in $\mathcal{C}_{\mathcal{M}}^{*}$ as defined in 1.7 is

$$
\left(y_{1}, \gamma^{1}\right) \otimes\left(y_{2}, \gamma^{2}\right)=\left(y_{1} y_{2},\left(\gamma^{1} \triangleleft y_{2}\right) \gamma^{2}\right) .
$$

This tensor product defines a group structure on the set of isomorphism classes of invertible objects

$$
\Lambda:=\bigcup_{y \in K}\{y\} \times \overline{F u n}_{y}
$$

by the equation $\left(y_{1},\left[\gamma^{1}\right]\right) \star\left(y_{2},\left[\gamma^{2}\right]\right)=\left(y_{1} y_{2},\left[\left(\gamma^{1} \triangleleft y_{2}\right) \gamma^{2}\right]\right)$ where $[\gamma]$ denotes the equivalence class of $\gamma$ in Fun .

Define the element $\gamma \in C^{1}\left(K, C^{1}\left(G, \operatorname{Map}\left(K, \mathbb{C}^{*}\right)\right)\right)$ by the equation

$$
\gamma(y):=\gamma_{y}
$$

and note that the equations $\delta_{G} \gamma_{y}=\frac{\mu \triangleleft y}{\mu}$ are equivalent to the equation

$$
\delta_{G} \gamma=\delta_{K} \mu \text {. }
$$


Define the element $\tilde{\nu}:=\delta_{K} \gamma$, i.e. $\tilde{\nu}\left(y_{1}, y_{2}\right)=\frac{\gamma\left(y_{2}\right) \gamma\left(y_{1}\right) \triangleleft y_{2}}{\gamma\left(y_{1} y_{2}\right)}$, and note that

$$
\delta_{K} \tilde{\nu}=\delta_{K}^{2} \gamma=1 \text { and } \delta_{G} \tilde{\nu}=\delta_{G} \delta_{K} \gamma=\delta_{K} \delta_{G} \gamma=\delta_{K}^{2} \mu=1 .
$$

Hence $\tilde{\nu} \in Z^{2}\left(K, Z^{1}\left(G, \operatorname{Map}\left(K, \mathbb{C}^{*}\right)\right)\right)$ and we may define

$$
\nu:=\psi \circ \tilde{\nu} \in Z^{2}\left(K, Z^{1}\left(A, \mathbb{C}^{*}\right)\right)=Z^{2}(K, \mathbb{A})
$$

thus having $\nu\left(y_{1}, y_{2}\right)(a):=\tilde{\nu}\left(y_{1}, y_{2}\right)(1 ; a)$.

With this 2 -cocycle $\nu$ we may define the crossed product $K \ltimes_{\nu} \mathbb{A}$ by setting on pairs of elements of the set $K \times \mathbb{A}$

$$
\left(y_{1}, \rho_{1}\right) \cdot\left(y_{2}, \rho_{2}\right):=\left(y_{1} y_{2}, \rho_{1}^{y_{2}} \rho_{2} \nu\left(y_{1}, y_{2}\right)\right) .
$$

Using the notation of (2.2) we have

Theorem 2.2. [10, Thm. 4.5] The map

$$
T: K \ltimes_{\nu} \mathbb{A} \rightarrow \Lambda, \quad T((y, \rho))=\left(y,\left[\zeta_{y}(\rho)\right]\right)
$$

is an isomorphism of groups. Hence $K_{0}\left(\mathcal{C}_{\mathcal{M}}^{*}\right) \cong \mathbb{Z}\left[K \ltimes_{\nu} \mathbb{A}\right]$.

Proof. On the one hand we have

$$
\begin{aligned}
T\left(\left(y_{1}, \rho_{1}\right) \cdot\left(y_{2}, \rho_{2}\right)\right) & =T\left(\left(y_{1} y_{2}, \rho_{1}^{y_{2}} \rho_{2} \nu\left(y_{1}, y_{2}\right)\right)\right) \\
& =\left(y_{1} y_{2},\left[\zeta_{y_{1}} y_{2}\left(\rho_{1}^{y_{2}} \rho_{2} \nu\left(y_{1}, y_{2}\right)\right)\right]\right)
\end{aligned}
$$

and on the other

$$
\begin{aligned}
T\left(\left(y_{1}, \rho_{1}\right)\right) \star T\left(\left(y_{2}, \rho_{2}\right)\right) & =\left(y_{1},\left[\zeta_{y_{1}}\left(\rho_{1}\right)\right]\right) \star\left(y_{2},\left[\zeta_{y_{2}}\left(\rho_{2}\right)\right]\right) \\
& =\left(y_{1} y_{2},\left[\left(\zeta_{y_{1}}\left(\rho_{1}\right) \triangleleft y_{2}\right) \zeta_{y_{2}}\left(\rho_{2}\right)\right]\right)
\end{aligned}
$$

The result follows if we check the equality

$$
\theta_{y_{1} y_{2}}\left(\left(\zeta_{y_{1}}\left(\rho_{1}\right) \triangleleft y_{2}\right) \zeta_{y_{2}}\left(\rho_{2}\right)\right)=\rho_{1}^{y_{2}} \rho_{2} \nu\left(y_{1}, y_{2}\right)
$$

since this implies that $\zeta_{y_{1} y_{2}}\left(\left(\rho_{1} \triangleleft y_{2}\right) \rho_{2} \nu\left(y_{1}, y_{2}\right)\right)$ and $\left(\zeta_{y_{1}}\left(\rho_{1}\right) \triangleleft y_{2}\right) \zeta_{y_{2}}\left(\rho_{2}\right)$ are cohomologous; hence we have

$$
\begin{aligned}
\theta_{y_{1} y_{2}}\left(\left(\zeta_{y_{1}}\left(\rho_{1}\right) \triangleleft y_{2}\right) \zeta_{y_{2}}\left(\rho_{2}\right)\right)(a) & =\frac{\left(\left(\zeta_{y_{1}}\left(\rho_{1}\right) \triangleleft y_{2}\right)(1 ; a)\right) \zeta_{y_{2}}\left(\rho_{2}\right)(1 ; a)}{\gamma\left(y_{1} y_{2}\right)(1 ; a)} \\
& =\frac{\left(\gamma\left(y_{1}\right) \triangleleft y_{2} \varphi\left(\rho_{1}\right) \triangleleft y_{2}\right)(1 ; a)\left(\gamma\left(y_{2}\right) \varphi\left(\rho_{2}\right)\right)(1 ; a)}{\gamma\left(y_{1} y_{2}\right)(1 ; a)} \\
& =\delta_{K} \gamma\left(y_{1}, y_{2}\right)(1 ; a) \rho_{1}^{y_{2}}(a) \rho_{2}(a) \\
& =\left(\nu\left(y_{1}, y_{2}\right) \rho_{1}^{y_{2}} \rho_{2}\right)(a) .
\end{aligned}
$$

2.3. A skeleton of the pointed category $\mathcal{C}_{\mathcal{M}}^{*}$. A skeleton $s k\left(\mathcal{C}_{\mathcal{M}}^{*}\right)$ of $\mathcal{C}_{\mathcal{M}}^{*}$ is a full subcategory of $\mathcal{C}_{\mathcal{M}}^{*}$ on which each object of $\mathcal{C}_{\mathcal{M}}^{*}$ is isomorphic to only one object in $\operatorname{sk}\left(\mathcal{C}_{\mathcal{M}}^{*}\right)$. Let us choose for objects

$$
o b\left(s k\left(\mathcal{C}_{\mathcal{M}}^{*}\right)\right):=\left\{\left(y, \zeta_{y}(\rho)\right) \mid(y, \rho) \in K \ltimes_{\nu} \mathbb{A}\right\}
$$

and define its tensor product $\bullet$ by the one induced by $\star$, i.e.

$$
\left(\left(y_{1}, \zeta_{y_{1}}\left(\rho_{1}\right)\right) \bullet\left(y_{2}, \zeta_{y_{2}}\left(\rho_{2}\right)\right):=\left(y_{1} y_{2}, \zeta_{y_{1} y_{2}}\left(\nu\left(y_{1}, y_{2}\right) \rho_{1}^{y_{2}} \rho_{1}\right)\right)\right. \text {. }
$$

For each pair of objects, choose isomorphisms in $\mathcal{C}_{\mathcal{M}}^{*}$

$$
f\left(\left(y_{1}, \zeta_{y_{1}}\left(\rho_{1}\right)\right),\left(y_{2}, \zeta_{y_{2}}\left(\rho_{2}\right)\right):\left(y_{1}, \zeta_{y_{1}}\left(\rho_{1}\right)\right) \bullet\left(y_{2}, \zeta_{y_{2}}\left(\rho_{2}\right)\right) \stackrel{\sim}{\rightarrow}\left(y_{1}, \zeta_{y_{1}}\left(\rho_{1}\right)\right) \otimes\left(y_{2}, \zeta_{y_{2}}\left(\rho_{2}\right)\right)\right.
$$


which by equation (2.1) satisfy

$$
\begin{aligned}
\left(\left(\zeta_{y_{1}}\left(\rho_{1}\right) \triangleleft y_{2}\right) \zeta_{y_{1}}\left(\rho_{1}\right)\right)(k ; g)=\frac{f\left(\left(y_{1}, \zeta_{y_{1}}\left(\rho_{1}\right)\right),\left(y_{2}, \zeta_{y_{2}}\left(\rho_{2}\right)\right)(k \triangleleft g)\right.}{f\left(\left(y_{1}, \zeta_{y_{1}}\left(\rho_{1}\right)\right),\left(y_{2}, \zeta_{y_{2}}\left(\rho_{2}\right)\right)(k)\right.} & \quad \times \zeta_{y_{1} y_{2}}\left(\nu\left(y_{1}, y_{2}\right) \rho_{1}^{y_{2}} \rho_{1}\right)(k ; g) .
\end{aligned}
$$

The tensor product $\otimes$ in $\mathcal{C}_{\mathcal{M}}^{*}$ is associative since it is defined by the composition of functors, but the tensor product $\bullet$ in its skeleton $\operatorname{sk}\left(\mathcal{C}_{\mathcal{M}}^{*}\right)$ may fail to be associative. The associativity constraint for $\operatorname{sk}\left(\mathcal{C}_{\mathcal{M}}^{*}\right)$ is then

$$
\begin{aligned}
\widehat{\omega}^{\prime}\left(\left(y_{1}, \zeta_{y_{1}}\left(\rho_{1}\right)\right),\left(y_{2}, \zeta_{y_{2}}\left(\rho_{2}\right)\right),\left(y_{3}, \zeta_{y_{3}}\left(\rho_{3}\right)\right)\right) \\
=\frac{f\left(\left(y_{1}, \zeta_{y_{1}}\left(\rho_{1}\right)\right),\left(y_{2}, \zeta_{y_{2}}\left(\rho_{2}\right)\right) \otimes I d_{\left(\zeta_{y_{3}}\left(\rho_{3}\right), y_{3}\right)}\right.}{f\left(\left(y_{1}, \zeta_{y_{1}}\left(\rho_{1}\right)\right),\left(y_{2}, \zeta_{y_{2}}\left(\rho_{2}\right)\right) \bullet\left(y_{3}, \zeta_{y_{3}}\left(\rho_{3}\right)\right)\right)} \\
\times \frac{f\left(\left(y_{1}, \zeta_{y_{1}}\left(\rho_{1}\right)\right) \bullet\left(y_{2}, \zeta_{y_{2}}\left(\rho_{2}\right)\right),\left(y_{3}, \zeta_{y_{3}}\left(\rho_{3}\right)\right)\right)}{I d_{\left(\zeta_{y_{1}}\left(\rho_{1}\right), y_{1}\right)} \otimes f\left(\left(y_{2}, \zeta_{y_{2}}\left(\rho_{2}\right)\right),\left(y_{3}, \zeta_{y_{3}}\left(\rho_{3}\right)\right)\right) .}
\end{aligned}
$$

In [10, Thm. 4.9] it is shown that $\widehat{\omega}^{\prime}$ is $K$-invariant and moreover that it can be given in explicit form by the equation

$$
\widehat{\omega}^{\prime}\left(\left(y_{1}, \zeta_{y_{1}}\left(\rho_{1}\right)\right),\left(y_{2}, \zeta_{y_{2}}\left(\rho_{2}\right)\right),\left(y_{3}, \zeta_{y_{3}}\left(\rho_{3}\right)\right)\right)=\tilde{\nu}\left(y_{1}, y_{2}\right)\left(1 ; u\left(y_{3}\right)\right) \rho_{1}\left(\kappa_{y_{2}, u\left(y_{3}\right)}\right) .
$$

Therefore we may define the 3-cocycle on $K \ltimes_{\nu} \mathbb{A}$ by the equation

$$
\widehat{\omega}\left(\left(y_{1}, \rho_{1}\right),\left(y_{2}, \rho_{2}\right),\left(y_{3}, \rho_{3}\right)\right)=\tilde{\nu}\left(y_{1}, y_{2}\right)\left(1 ; u\left(y_{3}\right)\right) \rho_{1}\left(\kappa_{y_{2}, u\left(y_{3}\right)}\right),
$$

and choosing $G=A \rtimes_{F} K$ and $u(y)=(1, y)$ as it was done at the end of 1.1 , the 3-cocycle on $K \ltimes_{\nu} \mathbb{A}$ becomes

$$
\widehat{\omega}\left(\left(y_{1}, \rho_{1}\right),\left(y_{2}, \rho_{2}\right),\left(y_{3}, \rho_{3}\right)\right)=\tilde{\nu}\left(y_{1}, y_{2}\right)\left(1 ;\left(1, y_{3}\right)\right) \rho_{1}\left(F\left(y_{2}, y_{3}\right)\right) .
$$

Therefore the skeleton $\operatorname{sk}\left(\mathcal{C}_{\mathcal{M}}^{*}\right)$ of $\mathcal{C}_{\mathcal{M}}^{*}$ becomes isomorphic to $\mathcal{V}\left(K \ltimes_{\nu} \mathbb{A}, \widehat{\omega}\right)$ which is equivalent to $\operatorname{Vect}\left(K \ltimes_{\nu} \mathbb{A}, \widehat{\omega}\right)$. Therefore we can conclude with

Theorem 2.3. [10, Thm. 4.9] The fusion categories $\mathcal{C}_{\mathcal{M}}^{*}=\mathcal{V}(G, \omega)_{\mathcal{M}(A \backslash G, \mu)}^{*}$ and $\operatorname{Vect}\left(K \ltimes_{\nu} \mathbb{A}, \widehat{\omega}\right)$ are equivalent.

Applying the results of $\$ 1.9$ we have

Corollary 2.4. The categories $\operatorname{Vect}\left(A \rtimes_{F} K, \omega\right)$ and $\operatorname{Vect}\left(K \ltimes_{\nu} \mathbb{A}, \widehat{\omega}\right)$ are weakly Morita equivalent. Hence their centers are are canonically equivalent

$$
\mathcal{Z}\left(V e c t\left(A \rtimes_{F} K, \omega\right)\right) \simeq \mathcal{Z}\left(V e c t\left(K \ltimes_{\nu} \mathbb{A}, \widehat{\omega}\right)\right)
$$

as braided tensor categories

\section{Weak Morita equivalence Classes of group-theoretical tensor CATEGORIES}

We are interested in classifying group theoretical tensor categories of a specific global dimension up to weak Morita equivalence. For this purpose we will fix the group $G=A \rtimes_{F} K$ with $A$ abelian and normal in $G$ and $F \in Z^{2}(K, A)$, and we will give an explicit description of the cocycles $\omega \in Z^{3}\left(A \rtimes_{F} K, \mathbb{C}^{*}\right)$ and $\widehat{\omega} \in Z^{3}\left(K \ltimes_{\nu} \mathbb{A}, \mathbb{C}^{*}\right)$ such that the tensor categories $\mathcal{V}\left(A \rtimes_{F} K, \omega\right)$ and $\mathcal{V}\left(K \ltimes_{\nu} \mathbb{A}, \widehat{\omega}\right)$ are weakly Morita equivalent. 
3.1. Description of $\omega, \mu$ and $\gamma$. In Theorem 2.1 and in $\$ 2.2$ we have seen the conditions needed for the tensor category $\mathcal{C}_{\mathcal{M}}^{*}=\mathcal{V}(G, \omega)_{\mathcal{M}(A \backslash G, \mu)}^{*}$ to be pointed. In particular we have seen that we need the existence of $\gamma \in C^{1}\left(K, C^{1}\left(G, \operatorname{Map}\left(K, \mathbb{C}^{*}\right)\right)\right)$ such that

$$
\delta_{G} \gamma=\delta_{K} \mu .
$$

Since we also have that $\delta_{G} \mu^{-1}=\pi^{*} \omega$ we can obtain the following lemma.

Lemma 3.1. The cocycles $\pi^{*} \omega$ and $\tilde{\nu}$ are cohomologous in $\operatorname{Tot}\left(C^{*}\left(K, C^{*}\left(G, \operatorname{Map}\left(K, \mathbb{C}^{*}\right)\right)\right)\right)$.

Proof. Recall the definition of the double complex $C^{*}\left(K, C^{*}\left(G, \operatorname{Map}\left(K, \mathbb{C}^{*}\right)\right)\right)$ given in $₫ 1.2 .1$, and note that we have $\pi^{*} \omega \in C^{0,3}, \mu \in C^{0,2}, \gamma \in C^{1,1}$ and $\tilde{\nu}=\delta_{K} \gamma \in C^{2,1}$, satisfying $\pi^{*} \omega \cdot \delta_{G} \mu=1$ and $\delta_{K} \mu \cdot \delta_{G} \gamma^{-1}=1$.

Consider the element $\mu \oplus \gamma \in \operatorname{Tot}^{2}$ and note that

$$
\delta_{T o t}(\mu \oplus \gamma)=\left(\delta_{K} \oplus \delta_{G}^{(-1)^{p}}\right)(\mu \oplus \gamma)=\delta_{G} \mu \oplus \delta_{K} \mu \cdot \delta_{G} \gamma^{-1} \oplus \delta_{K} \gamma
$$

Therefore

$$
\pi^{*} \omega \cdot \delta_{T o t}(\mu \oplus \gamma)=\tilde{\nu}
$$

Lemma 3.1 implies further conditions on the cohomology class of $\omega$ for the tensor category $\mathcal{C}_{\mathcal{M}}^{*}=\mathcal{V}(G, \omega)_{\mathcal{M}(A \backslash G, \mu)}^{*}$ to be pointed.

Corollary 3.2. If the tensor category $\mathcal{C}_{\mathcal{M}}^{*}=\mathcal{V}(G, \omega)_{\mathcal{M}(A \backslash G, \mu)}^{*}$ is pointed then $\omega$ is cohomologous to a cocycle that lives in $C^{2,1} \oplus C^{3,0}$ of the double complex that induces the Lyndon-Hochschild-Serre spectral sequence.

Remark 3.3. Note that this implies that the cohomology class of $\omega$ belongs to the subgroup of $H^{3}\left(G, \mathbb{C}^{*}\right)$ defined as

$$
\Omega(G ; A):=\operatorname{ker}\left(\operatorname{ker}\left(H^{3}\left(G, \mathbb{C}^{*}\right) \rightarrow E_{\infty}^{0,3}\right) \rightarrow E_{\infty}^{1,2}\right)
$$

which fits into the short exact sequence

$$
1 \rightarrow E_{\infty}^{3,0} \rightarrow \Omega(G ; A) \rightarrow E_{\infty}^{2,1} \rightarrow 1 .
$$

The cohomology classes in $\Omega(G ; A)$ are the only cohomology classes such that $\mathcal{C}_{\mathcal{M}}^{*}=\mathcal{V}(G, \omega)_{\mathcal{M}(A \backslash G, \mu)}^{*}$ is pointed.

In what follows we will construct explicit representatives for $\omega$ and $\mu$, but for this purpose we will start by constructing explicit 3-cocyles in $\operatorname{Tot}\left(C^{*}\left(K, C^{*}\left(G, \operatorname{Map}\left(K, \mathbb{C}^{*}\right)\right)\right)\right)$ which appear in $\Omega(G ; A)$. Let us start by determining the second differential $d_{2}: E_{2}^{2,1} \rightarrow E_{2}^{4,0}$.

Lemma 3.4. The second differential $d_{2}: E_{2}^{2,1} \rightarrow E_{2}^{4,0}$ is isomorphic to the homomorphism

$$
\begin{aligned}
H^{2}(K, \mathbb{A}) & \rightarrow H^{4}\left(K, \mathbb{C}^{*}\right), \quad[\widehat{F}] \mapsto\left[(\widehat{F} \wedge F)^{-1}\right] \\
\text { where }(\widehat{F} \wedge F)\left(k_{1}, k_{2}, k_{3}, k_{4}\right) & :=\widehat{F}\left(k_{1}, k_{2}\right)\left(F\left(k_{3}, k_{4}\right)\right) .
\end{aligned}
$$

Proof. First recall that

$$
\begin{aligned}
& E_{2}^{2,1}=H^{2}\left(K, H^{1}\left(G, \operatorname{Map}\left(K, \mathbb{C}^{*}\right)\right)\right) \cong H^{2}\left(K, \operatorname{Hom}\left(A, \mathbb{C}^{*}\right)\right)=H^{2}(K, \mathbb{A}) \\
& E_{2}^{4,0}=H^{4}\left(K, H^{0}\left(G, \operatorname{Map}\left(K, \mathbb{C}^{*}\right)\right)\right)=H^{4}\left(K, \operatorname{Map}\left(K, \mathbb{C}^{*}\right)^{G}\right) \cong H^{4}\left(K, \mathbb{C}^{*}\right) .
\end{aligned}
$$


Take $\widehat{F} \in Z^{2}(K, \mathbb{A})$ and use the map $\varphi$ of Lemma 1.1 to lift this cocycle to $\varphi(\widehat{F}) \in C^{2}\left(K, Z^{1}\left(G, \operatorname{Map}\left(K, \mathbb{C}^{*}\right)\right)\right)$; in coordinates:

$$
\begin{aligned}
\varphi(\widehat{F})\left(k_{1}, k_{2}\right)\left(x_{1},\left(a_{2}, x_{2}\right)\right) & =\widehat{F}\left(k_{1}, k_{2}\right)\left(\kappa_{x_{1},\left(a_{2}, x_{2}\right)}\right)=\widehat{F}\left(k_{1}, k_{2}\right)\left({ }^{x_{1}} a_{2} F\left(x_{1}, x_{2}\right)\right) \\
& =\widehat{F}\left(k_{1}, k_{2}\right)\left({ }^{x_{1}} a_{2}\right) \widehat{F}\left(k_{1}, k_{2}\right)\left(F\left(x_{1}, x_{2}\right)\right) .
\end{aligned}
$$

Its boundary is

$$
\begin{aligned}
\delta_{k} \varphi(\widehat{F})\left(k_{1}, k_{2}, k_{3}\right) & \left(x_{1},\left(a_{2}, x_{2}\right)\right) \\
= & \widehat{F}\left(k_{2}, k_{3}\right)\left({ }^{x_{1}} a_{2} F\left(x_{1}, x_{2}\right)\right) \widehat{F}\left(k_{1} k_{2}, k_{3}\right)\left({ }^{x_{1}} a_{2} F\left(x_{1}, x_{2}\right)\right)^{-1} \\
& \widehat{F}\left(k_{1}, k_{2} k_{3}\right)\left({ }^{x_{1}} a_{2} F\left(x_{1}, x_{2}\right)\right) \widehat{F}\left(k_{1}, k_{2}\right)\left({ }^{k_{3} x_{1}} a_{2} F\left(k_{3} x_{1}, x_{2}\right)\right)^{-1} \\
= & \widehat{F}\left(k_{1}, k_{2}\right){ }^{k_{3}}\left(F\left(x_{1}, x_{2}\right)\right) \widehat{F}\left(k_{1}, k_{2}\right)\left(F\left(k_{3} x_{1}, x_{2}\right)\right)^{-1} \\
= & \widehat{F}\left(k_{1}, k_{2}\right)\left(\frac{F\left(k_{3}, x_{1}\right)}{F\left(k_{3}, x_{1} x_{2}\right)}\right),
\end{aligned}
$$

and we can define $u \in C^{3}\left(K, C^{0}\left(G, \operatorname{Map}\left(K, \mathbb{C}^{*}\right)\right)\right)$ as

$$
u\left(k_{1}, k_{2}, k_{3}\right)(x):=\widehat{F}\left(k_{1}, k_{2}\right)\left(F\left(k_{3}, x\right)\right) .
$$

On the one hand we have

$$
\begin{aligned}
\delta_{G} u\left(k_{1}, k_{2}, k_{3}\right)\left(x_{1},\left(a_{2}, x_{2}\right)\right) & =u\left(k_{1}, k_{2}, k_{3}\right)\left(x_{1} x_{2}\right) u\left(k_{1}, k_{2}, k_{3}\right)\left(x_{1}\right)^{-1} \\
& =\widehat{F}\left(k_{1}, k_{2}\right)\left(\frac{F\left(k_{3}, x_{1} x_{2}\right)}{F\left(k_{3}, x_{1}\right)}\right)
\end{aligned}
$$

and on the other

$$
\begin{aligned}
& \delta_{K} u\left(k_{1}, k_{2}, k_{3}, k_{4}\right)(x) \\
&= \widehat{F}\left(k_{2}, k_{3}\right)\left(F\left(k_{4}, x\right)\right) \widehat{F}\left(k_{1} k_{2}, k_{3}\right)\left(F\left(k_{4}, x\right)\right)^{-1} \widehat{F}\left(k_{1}, k_{2} k_{3}\right)\left(F\left(k_{4}, x\right)\right) \\
& \widehat{F}\left(k_{1}, k_{2}\right)\left(F\left(k_{3} k_{4}, x\right)\right)^{-1} \widehat{F}\left(k_{1}, k_{2}\right)\left(F\left(k_{3}, k_{4} x\right)\right) \\
&= \widehat{F}\left(k_{1}, k_{2}\right)^{k_{3}}\left(F\left(k_{4}, x\right)\right) \widehat{F}\left(k_{1}, k_{2}\right)\left(F\left(k_{3} k_{4}, x\right)\right)^{-1} \widehat{F}\left(k_{1}, k_{2}\right)\left(F\left(k_{3}, k_{4} x\right)\right) \\
&= \widehat{F}\left(k_{1}, k_{2}\right)\left(F\left(k_{3}, k_{4}\right)\right) .
\end{aligned}
$$

Since $\delta_{G} u=\delta_{K} \varphi(\widehat{F})$ we have that

$$
\delta_{T o t}\left(\varphi(\widehat{F}) \oplus u^{-1}\right)=\delta_{K} \varphi(\widehat{F}) \delta_{G} u \oplus \delta_{k} u^{-1}=(\widehat{F} \wedge F)^{-1} ;
$$

therefore $d_{2}[\varphi(\widehat{F})]=\left[(\widehat{F} \wedge F)^{-1}\right]$.

Suppose that $d_{2}[\varphi(\widehat{F})]=0$, hence there exists $\epsilon \in C^{3}\left(K, \mathbb{C}^{*}\right)$ such that $\delta_{K} \epsilon=$ $\widehat{F} \wedge F$. Define $\bar{\epsilon} \in C^{3}\left(K, C^{0}\left(G, \operatorname{Maps}\left(K, \mathbb{C}^{*}\right)\right)\right)$ by the equation

$$
\bar{\epsilon}\left(k_{1}, k_{2}, k_{3}\right)(x):=\epsilon\left(k_{1}, k_{2}, k_{3}\right)
$$

and note that $\delta_{K} \bar{\epsilon}=\widehat{F} \wedge F$ and that $\delta_{G} \bar{\epsilon}=1$. Hence the class $\varphi(\widehat{F}) \oplus \bar{\epsilon} u^{-1} \epsilon$ $C^{2,1} \oplus C^{3,0}$ defines a 3 -cocycle in the total complex:

$$
\varphi(\widehat{F}) \oplus \bar{\epsilon} u^{-1} \in Z^{3} \operatorname{Tot}\left(C^{*}\left(K, C^{*}\left(G, \operatorname{Map}\left(K, \mathbb{C}^{*}\right)\right)\right)\right) .
$$

Define $\beta \in C^{2}\left(K, C^{0}\left(G, \operatorname{Maps}\left(K, \mathbb{C}^{*}\right)\right)\right)$ by the equation

$$
\beta\left(k_{1}, k_{2}\right)(x):=\epsilon\left(k_{1}, k_{2}, x\right)
$$


and note that

$$
\begin{aligned}
\delta_{K} \beta\left(k_{1}, k_{2}, k_{3}\right)(x) & =\epsilon\left(k_{2}, k_{3}, x\right) \epsilon\left(k_{1} k_{2}, k_{3}, x\right)^{-1} \epsilon\left(k_{1}, k_{2} k_{3}, x\right) \epsilon\left(k_{1}, k_{2}, k_{3} x\right)^{-1} \\
& =\delta_{K} \epsilon\left(k_{1}, k_{2}, k_{3}, x\right) \epsilon\left(k_{1}, k_{2}, k_{3}\right)^{-1} \\
& =\widehat{F}\left(k_{1}, k_{2}\right)\left(\left(F\left(k_{3}, x\right)\right) \bar{\epsilon}\left(k_{1}, k_{2}, k_{3}\right)(x)^{-1} .\right.
\end{aligned}
$$

Therefore $\delta_{K} \beta \bar{\epsilon} u^{-1}=1$, hence we have that the class $\varphi(\widehat{F}) \delta_{G} \beta \in C^{2,1}$ is a 3cocycle in the total complex and moreover that it is cohomologous to the class $\varphi(\widehat{F}) \oplus \bar{\epsilon} u^{-1}$, in coordinates:

$$
\begin{gathered}
\left(\varphi(\widehat{F}) \delta_{G} \beta\right)\left(k_{1}, k_{2}\right)\left(x_{1},\left(a_{2}, x_{2}\right)\right)=\widehat{F}\left(k_{1}, k_{2}\right)\left({ }^{x_{1}} a_{2}\right) \widehat{F}\left(k_{1}, k_{2}\right)\left(F\left(x_{1}, x_{2}\right)\right) \\
\\
\epsilon\left(k_{1}, k_{2}, x_{1} x_{2}\right) \epsilon\left(k_{1}, k_{2}, x_{1}\right)^{-1} .
\end{gathered}
$$

Summarizing the previous results:

Proposition 3.5. Every cohomology class which appears in $\Omega(G ; A)$ can be represented by a 3-cocycle $\varphi(\widehat{F}) \delta_{G} \beta \in C^{2,1}$ with $\widehat{F} \in Z^{2}(K, \mathbb{A}), \beta\left(k_{1}, k_{2}\right)(x)=\epsilon^{\prime}\left(k_{1}, k_{2}, x\right)$ and $\delta_{K} \epsilon^{\prime}=\widehat{F} \wedge F$.

Proof. Take $[\omega] \in \Omega(G ; A)$ and let $[\widehat{F}] \in E_{2}^{2,1}$ be a representative of the cohomology class of the image of $[\omega]$ in $E_{\infty}^{2,1}$. Since $d_{2}[\varphi(\widehat{F})]=0$ we know that the cohomology class $\left[\varphi(\widehat{F}) \oplus \bar{\epsilon} u^{-1}\right]$ constructed above belongs to $\Omega(G ; A)$. Therefore we have that

$$
\left[\omega^{-1}\right] \cdot\left[\varphi(\widehat{F}) \oplus \bar{\epsilon} u^{-1}\right] \in E_{\infty}^{3,0}
$$

and hence we can choose a representative cocycle $[\tau] \in H^{3}\left(K, \mathbb{C}^{*}\right) \cong E_{2}^{3,0}$ such that

$$
[\omega]=\left[\varphi(\widehat{F}) \oplus \bar{\epsilon} \bar{\tau} u^{-1}\right]
$$

with $\bar{\tau} \in C^{3}\left(K, C^{0}\left(G, \operatorname{Maps}\left(K, \mathbb{C}^{*}\right)\right)\right)$ defined as

$$
\bar{\tau}\left(k_{1}, k_{2}, k_{3}\right)(x):=\tau\left(k_{1}, k_{2}, k_{3}\right) .
$$

Let $\epsilon^{\prime}:=\epsilon \tau$ and define $\beta \in C^{2}\left(K, C^{0}\left(G, \operatorname{Maps}\left(K, \mathbb{C}^{*}\right)\right)\right)$ by the equation

$$
\beta\left(k_{1}, k_{2}\right)(x):=\epsilon^{\prime}\left(k_{1}, k_{2}, x\right) .
$$

Equation (3.1) implies that $\delta_{K} \beta=(\bar{\epsilon} \bar{\tau})^{-1} u$ and therefore the proposition follows from the equation

$$
\left(\varphi(\widehat{F}) \oplus \bar{\epsilon} \bar{\tau} u^{-1}\right) \delta_{T o t} \beta=\varphi(\widehat{F}) \delta_{G} \beta \oplus \delta_{K} \beta \bar{\epsilon} \bar{\tau} u^{-1}=\varphi(\widehat{F}) \delta_{G} \beta .
$$

Now we need to find an explicit description of $\omega \in Z^{3}\left(G, \mathbb{C}^{*}\right)$ such that $\pi^{*} \omega$ and $\varphi(\widehat{F}) \delta_{G} \beta$ are cohomologous.

Theorem 3.6. Let $G=A \rtimes_{F} K$ and consider $\omega \in C^{3}\left(G, \mathbb{C}^{*}\right), \mu \in C^{0,2}$ and $\gamma \in C^{1,1}$ defined by the following equations:

$$
\begin{aligned}
\omega\left(\left(a_{1}, x_{1}\right),\left(a_{2}, x_{2}\right),\left(a_{3}, x_{3}\right)\right) & :=\widehat{F}\left(x_{1}, x_{2}\right)\left(a_{3}\right) \epsilon\left(x_{1}, x_{2}, x_{3}\right) \\
\mu\left(x_{1},\left(a_{2}, x_{2}\right),\left(a_{3}, x_{3}\right)\right) & =\left(\widehat{F}\left(x_{1}, x_{2}\right)\left(a_{3}\right) \epsilon\left(x_{1}, x_{2}, x_{3}\right)\right)^{-1} \\
\gamma(y)\left(x_{1},\left(a_{2}, x_{2}\right)\right) & =\widehat{F}\left(y, x_{1}\right)\left(a_{2}\right) \epsilon\left(y, x_{1}, x_{2},\right) .
\end{aligned}
$$

Then $\pi^{*} \omega \cdot\left(\delta_{\text {Tot }} \mu \oplus \gamma\right)=\varphi(\widehat{F}) \delta_{G} \beta$. 
Proof. Let us calculate:

$$
\begin{aligned}
\delta_{G} \mu\left(x_{1},\right. & \left.\left(a_{2}, x_{2}\right),\left(a_{3}, x_{3}\right),\left(a_{4}, x_{4}\right)\right) \\
= & \mu\left(x_{1} x_{2},\left(a_{3}, x_{3}\right),\left(a_{4}, x_{4}\right)\right) \mu\left(x_{1},\left(a_{2}{ }^{x_{2}} a_{3} F\left(x_{2}, x_{3}\right), x_{2} x_{3}\right),\left(a_{3}, x_{3}\right)\right)^{-1} \\
& \mu\left(x_{1},\left(a_{2}, x_{2}\right)\left(a_{3}{ }^{x_{3}} a_{4} F\left(x_{3}, x_{4}\right), x_{3} x_{4}\right)\right) \mu\left(x_{1},\left(a_{2}, x_{2}\right),\left(a_{3}, x_{3}\right)\right)^{-1} \\
= & \widehat{F}\left(x_{1} x_{2}, x_{3}\right)\left(a_{4}\right)^{-1} \widehat{F}\left(x_{1}, x_{2} x_{3}\right)\left(a_{4}\right) \widehat{F}\left(x_{1}, x_{2}\right)\left(a_{3}{ }^{x_{3}} a_{4} F\left(x_{3}, x_{4}\right)\right)^{-1} \\
& \widehat{F}\left(x_{1}, x_{2}\right)\left(a_{3}\right) \epsilon\left(x_{2}, x_{3}, x_{4}\right)^{-1} \delta_{K} \epsilon\left(x_{1}, x_{2}, x_{3}, x_{4}\right) \\
= & \widehat{F}\left(x_{2}, x_{3}\right)\left(a_{4}\right)^{-1} \epsilon\left(x_{2}, x_{3}, x_{4}\right)^{-1},
\end{aligned}
$$

and

$$
\begin{aligned}
\pi^{*} \omega\left(x_{1},\left(a_{2}, x_{2}\right),\left(a_{3}, x_{3}\right),\left(a_{4}, x_{4}\right)\right) & =\omega\left(\left(a_{2}, x_{2}\right),\left(a_{3}, x_{3}\right),\left(a_{4}, x_{4}\right)\right) \\
& =\widehat{F}\left(x_{2}, x_{3}\right)\left(a_{4}\right) \epsilon\left(x_{2}, x_{3}, x_{4}\right),
\end{aligned}
$$

hence we have that

$$
\delta_{G} \mu \cdot \pi^{*} \omega=1 .
$$

Now

$$
\begin{aligned}
\delta_{K} \mu(y)\left(x_{1},\left(a_{2}, x_{2}\right),\left(a_{3}, x_{3}\right)\right) & \\
& =\mu\left(x_{1},\left(a_{2}, x_{2}\right),\left(a_{3}, x_{3}\right)\right) \mu\left(y x_{1},\left(a_{2}, x_{2}\right),\left(a_{3}, x_{3}\right)\right)^{-1} \\
& =\frac{\widehat{F}\left(y x_{1}, x_{2}\right)\left(a_{3}\right) \epsilon\left(y x_{1}, x_{2}, x_{3}\right)}{\widehat{F}\left(x_{1}, x_{2}\right)\left(a_{3}\right) \epsilon\left(x_{1}, x_{2}, x_{3}\right)},
\end{aligned}
$$

and

$$
\begin{aligned}
\delta_{G} \gamma & (y)\left(x_{1},\left(a_{2}, x_{2}\right),\left(a_{3}, x_{3}\right)\right) \\
= & \gamma(y)\left(x_{1} x_{2},\left(a_{3}, x_{3}\right)\right) \gamma(y)\left(x_{1},\left(a_{2}{ }^{x_{2}} a_{3} F\left(x_{2}, x_{3}\right), x_{2} x_{3}\right)\right)^{-1} \gamma(y)\left(x_{1},\left(a_{2}, x_{2}\right)\right) \\
= & \widehat{F}\left(y, x_{1} x_{2}\right)\left(a_{3}\right) \widehat{F}\left(y, x_{1}\right)\left(a_{2}{ }^{x_{2}} a_{3} F\left(x_{2}, x_{3}\right)\right)^{-1} \widehat{F}\left(y, x_{1}\right)\left(a_{2}\right) \\
& \epsilon\left(y, x_{1} x_{2}, x_{3}\right) \epsilon\left(y, x_{1}, x_{2} x_{3}\right)^{-1} \epsilon\left(y, x_{1}, x_{2}\right) \\
= & \widehat{F}\left(y x_{1}, x_{2}\right)\left(a_{3}\right) \widehat{F}\left(x_{1}, x_{2}\right)\left(a_{3}\right)^{-1} \epsilon\left(y x_{1}, x_{2}, x_{3}\right) \epsilon\left(x_{1}, x_{2}, x_{3}\right)^{-1},
\end{aligned}
$$

hence we have that

$$
\delta_{K} \mu \cdot \delta_{G} \gamma^{-1}=1
$$

Finally we calculate

$$
\begin{aligned}
\delta_{K} \gamma\left(k_{1}, k_{2}\right) & \left(x_{1},\left(a_{2}, x_{2}\right)\right) \\
= & \gamma\left(k_{2}\right)\left(x_{1},\left(a_{2}, x_{2}\right)\right) \gamma\left(k_{1} k_{2}\right)\left(x_{1},\left(a_{2}, x_{2}\right)\right)^{-1} \gamma\left(k_{1}\right)\left(k_{2} x_{1},\left(a_{2}, x_{2}\right)\right) \\
= & \widehat{F}\left(k_{2}, x_{1}\right)\left(a_{2}\right) \widehat{F}\left(k_{1} k_{2}, x_{1}\right)\left(a_{2}\right)^{-1} \widehat{F}\left(k_{1}, k_{2} x_{2}\right)\left(a_{2}\right) \\
& \epsilon\left(k_{2}, x_{1}, x_{2}\right) \epsilon\left(k_{1} k_{2}, x_{1}, x_{2}\right)^{-1} \epsilon\left(k_{1}, k_{2} x_{1}, x_{2}\right) \\
= & \widehat{F}\left(k_{1}, k_{2}\right)\left({ }^{x_{1}} a_{2}\right) \delta_{K} \epsilon\left(k_{1}, k_{2}, x_{1}, x_{2}\right) \epsilon\left(k_{1}, k_{2}, x_{1} x_{2}\right) \epsilon\left(k_{1}, k_{2}, x_{1}\right)^{-1} \\
= & \widehat{F}\left(k_{1}, k_{2}\right)\left({ }^{x_{1}} a_{2}\right) \widehat{F}\left(k_{1}, k_{2}\right)\left(F\left(x_{1}, x_{2}\right)\right) \epsilon\left(k_{1}, k_{2}, x_{1} x_{2}\right) \epsilon\left(k_{1}, k_{2}, x_{1}\right)^{-1},
\end{aligned}
$$

and since by equation (3.2) we have that

$$
\begin{gathered}
\left(\varphi(\widehat{F}) \delta_{G} \beta\right)\left(k_{1}, k_{2}\right)\left(x_{1},\left(a_{2}, x_{2}\right)\right)=\widehat{F}\left(k_{1}, k_{2}\right)\left({ }^{x_{1}} a_{2}\right) \widehat{F}\left(k_{1}, k_{2}\right)\left(F\left(x_{1}, x_{2}\right)\right) \\
\\
\epsilon\left(k_{1}, k_{2}, x_{1} x_{2}\right) \epsilon\left(k_{1}, k_{2}, x_{1}\right)^{-1}
\end{gathered}
$$


we have that

$$
\delta_{K} \gamma=\varphi(\widehat{F}) \delta_{G} \beta .
$$

Hence $\pi^{*} \omega \cdot\left(\delta_{\text {Tot }} \mu \oplus \gamma\right)=\varphi(\widehat{F}) \delta_{G} \beta$.

3.2. Description of $\widehat{\omega}$ and $\nu$. Assuming the explicit descriptions of $\omega, \mu$ and $\gamma$ described in Theorem 3.6, we see that $\tilde{\nu}=\varphi(\widehat{F}) \delta_{G} \beta$. Applying this explicit description of $\tilde{\nu}$ into the definition of $\nu$ given in (2.3) and of $\widehat{\omega}$ given in (2.4) we obtain

$$
\nu\left(k_{1}, k_{2}\right)(a):=\tilde{\nu}\left(k_{1}, k_{2}\right)(1,(a, 1))=\widehat{F}\left(k_{1}, k_{2}\right)(a)
$$

which implies that $\nu=\widehat{F}$, and

$$
\begin{aligned}
\widehat{\omega}\left(\left(k_{1}, \rho_{1}\right),\left(k_{2}, \rho_{2}\right),\left(k_{3}, \rho_{3}\right)\right): & =\tilde{\nu}\left(k_{1}, k_{2}\right)\left(1 ;\left(1, k_{3}\right)\right) \rho_{1}\left(F\left(k_{2}, k_{3}\right)\right) \\
& =\epsilon\left(k_{1}, k_{2}, k_{3}\right) \rho_{1}\left(F\left(k_{2}, k_{3}\right)\right) .
\end{aligned}
$$

After applying Corollary 2.4 to the previous explicit construction of $\widehat{\omega}$ we obtain the following theorem:

Theorem 3.7. Let $K$ be a finite group acting on the finite abelian group A. Consider cocycles $F \in Z^{2}(K, A)$ and $\widehat{F} \in Z^{2}(K, \mathbb{A})$ such that $\widehat{F} \wedge F$ is trivial in cohomology, i.e. there exists $\epsilon \in C^{3}\left(K, \mathbb{C}^{*}\right)$ such that $\delta_{K} \epsilon=\widehat{F} \wedge F$. Define the 3-cocycles $\omega \in Z^{3}\left(A \rtimes_{F} K, \mathbb{C}^{*}\right)$ and $\widehat{\omega} \in Z^{3}\left(K \ltimes_{\widehat{F}} \mathbb{A}, \mathbb{C}^{*}\right)$ by the equations:

$$
\begin{aligned}
& \omega\left(\left(a_{1}, k_{1}\right),\left(a_{2}, k_{2}\right),\left(a_{3}, k_{3}\right)\right):=\widehat{F}\left(k_{1}, k_{2}\right)\left(a_{3}\right) \epsilon\left(k_{1}, k_{2}, k_{3}\right) \\
& \widehat{\omega}\left(\left(k_{1}, \rho_{1}\right),\left(k_{2}, \rho_{2}\right),\left(k_{3}, \rho_{3}\right)\right):=\epsilon\left(k_{1}, k_{2}, k_{3}\right) \rho_{1}\left(F\left(k_{2}, k_{3}\right)\right) .
\end{aligned}
$$

Then the tensor categories $\operatorname{Vect}\left(A \rtimes_{F} K, \omega\right)$ and $\operatorname{Vect}\left(K \ltimes_{\widehat{F}} \mathbb{A}, \widehat{\omega}\right)$ are weakly Morita equivalent, and therefore their centers are braided equivalent

$$
\mathcal{Z}\left(\operatorname{Vect}\left(A \rtimes_{F} K, \omega\right)\right) \simeq \mathcal{Z}\left(\operatorname{Vect}\left(K \ltimes_{\widehat{F}} \mathbb{A}, \widehat{\omega}\right)\right) .
$$

Note that we may have taken a different choice of $\mu$ and $\gamma$ in section 3.1 thus producing different $\tilde{\nu}$ and $\widehat{\omega}$. The description of $\widehat{\omega}$ depends on the choice of cohomology class $[\widehat{F}] \in H^{2}(K, \mathbb{A}) \cong E_{2}^{2,1}$ in the second page representing the image of $[\omega]$ in $E_{3}^{2,1}=E_{\infty}^{2,1}$. This choice may be changed by elements in the image of the second differential $d_{2}: E_{2}^{0,2} \rightarrow E_{2}^{2,1}$.

Changing $\omega$ by a coboundary $\omega^{\prime}=\omega \delta_{G} \alpha$, and writing $\omega^{\prime}$ explicitly as

$$
\omega^{\prime}\left(\left(a_{1}, x_{1}\right),\left(a_{2}, x_{2}\right),\left(a_{3}, x_{3}\right)\right):=\widehat{F}^{\prime}\left(x_{1}, x_{2}\right)\left(a_{3}\right) \epsilon^{\prime}\left(x_{1}, x_{2}, x_{3}\right),
$$

produces a $\widehat{\omega}^{\prime}$ which becomes

$$
\widehat{\omega}^{\prime}\left(\left(k_{1}, \rho_{1}\right),\left(k_{2}, \rho_{2}\right),\left(k_{3}, \rho_{3}\right)\right):=\epsilon^{\prime}\left(k_{1}, k_{2}, k_{3}\right) \rho_{1}\left(F\left(k_{2}, k_{3}\right)\right) .
$$

Applying Theorem 3.7 and using the equivalence of categories $V e c t\left(A \rtimes_{F} K, \omega\right) \simeq$ $\operatorname{Vect}\left(A \rtimes_{F} K, \omega^{\prime}\right)$ we obtain that the tensor categories $V e c t\left(A \rtimes_{F} K, \omega\right)$ and $V e c t\left(K \ltimes_{\widehat{F}^{\prime}}\right.$ $\left.\mathbb{A}, \widehat{\omega}^{\prime}\right)$ are also weakly Morita equivalent. The previous argument permit us to conclude the following corollary:

Corollary 3.8. Suppose that the fusion category $\mathcal{C}_{\mathcal{M}}^{*}=\mathcal{V}\left(A \rtimes_{F} K, \omega\right)_{\mathcal{M}(K, \mu)}^{*}$ is pointed. Then it is equivalent to the category $\operatorname{Vect}\left(K \ltimes_{\widehat{F}^{\prime}} \mathbb{A}, \widehat{\omega}^{\prime}\right)$ where $\widehat{\omega}^{\prime}$ and $\omega^{\prime}$ are the cocycles defined in (3.3) and (3.4) respectively and $\omega^{\prime}$ is cohomologous to $\omega$. 
3.3. Classification theorem. Now we are ready to state the key result in order to establish the weak Morita equivalence classes of group theoretical tensor categories.

Theorem 3.9. Let $H$ and $\widehat{H}$ be finite groups, $\eta \in Z^{3}\left(H, \mathbb{C}^{*}\right)$ and $\widehat{\eta} \in Z^{3}\left(\widehat{H}, \mathbb{C}^{*}\right)$. Then the tensor categories $\operatorname{Vect}(H, \eta)$ and $\operatorname{Vect}(\widehat{H}, \widehat{\eta})$ are weakly Morita equivalent if and only if the following conditions are satified:

- There exist isomorphisms of groups

$$
\phi: G=A \rtimes_{F} K \stackrel{\cong}{\rightarrow} H \quad \widehat{\phi}: \widehat{G}=K \ltimes_{\widehat{F}} \mathbb{A} \stackrel{\cong}{\rightrightarrows} \widehat{H}
$$

for some finite group $K$ acting on the abelian group $A$, with $F \in Z^{2}(K, A)$ and $\widehat{F} \in Z^{2}(K, \mathbb{A})$ where $\mathbb{A}:=\operatorname{Hom}\left(A, \mathbb{C}^{*}\right)$.

- There exist $\epsilon: K^{3} \rightarrow \mathbb{C}^{*}$ such that $\widehat{F} \wedge F=\delta_{K} \epsilon$.

- The cohomology classes satisfy the equations $\left[\phi^{*} \eta\right]=[\omega]$ and $\left[\widehat{\phi}^{*} \widehat{\eta}\right]=[\widehat{\omega}]$ with

$$
\begin{aligned}
& \omega\left(\left(a_{1}, k_{1}\right),\left(a_{2}, k_{2}\right),\left(a_{3}, k_{3}\right)\right):=\widehat{F}\left(k_{1}, k_{2}\right)\left(a_{3}\right) \epsilon\left(k_{1}, k_{2}, k_{3}\right) \\
& \widehat{\omega}\left(\left(k_{1}, \rho_{1}\right),\left(k_{2}, \rho_{2}\right),\left(k_{3}, \rho_{3}\right)\right):=\epsilon\left(k_{1}, k_{2}, k_{3}\right) \rho_{1}\left(F\left(k_{2}, k_{3}\right)\right) .
\end{aligned}
$$

Proof. Suppose that $V \operatorname{ect}(H, \eta)$ and $V \operatorname{ect}(\widehat{H}, \widehat{\eta})$ are weaky Morita equivalent. Then $\operatorname{Vect}(\widehat{H}, \widehat{\eta})$ is equivalent to the dual category $\mathcal{V}(H, \eta)_{\mathcal{M}(A \backslash H, \mu)}^{*}$ with $K:=A \backslash H, \phi:$ $G=A \rtimes_{F} K \stackrel{\cong}{\rightrightarrows} H$ and $\mathcal{M}(A \backslash H, \mu)$ some module category of $\mathcal{V}(H, \eta)$. By Corollary 3.8 the tensor category $\operatorname{Vect}(\widehat{H}, \widehat{\eta})$ is furthermore equivalent to $\operatorname{Vect}\left(K \ltimes \widehat{F}^{\prime} \mathbb{A}, \widehat{\omega}^{\prime}\right)$ where $\omega^{\prime}$ and $\widehat{\omega}^{\prime}$ are the cocycles defined in equations (3.3) and (3.4) respectively, and such that $\omega^{\prime}$ is cohomologous to $\phi^{*} \eta$. In particular we have that $\widehat{\phi}: \widehat{G}=$ $K \ltimes_{\widehat{F}} \mathbb{A} \stackrel{\cong}{\rightrightarrows} \widehat{H}$ and that $\widehat{\phi}^{*} \widehat{\eta}$ is cohomologous to $\widehat{\omega}^{\prime}$.

The converse is the statement of Theorem 3.7 .

In the case that both $\omega$ and $\widehat{\omega}$ were cohomologically trivial, we conclude that $\operatorname{Vect}\left(A \rtimes_{F} K, 1\right)$ and $\operatorname{Vect}\left(K \ltimes_{\widehat{F}} \mathbb{A}, 1\right)$ are weakly Morita equivalent if and only if the cohomology class $[\widehat{F}] \in H^{2}(K, \mathbb{A})$ lies in the image of the second differential of the spectral sequence $d_{2}: H^{2}\left(A, \mathbb{C}^{*}\right)^{K} \rightarrow H^{2}(K, \mathbb{A})$. This result was originally proved in [3, Cor. 6.2].

\section{ExAmples}

4.1. Pointed fusion categories of global dimension 4. We can now calculate the weakly Morita equivalence classes of pointed fusion categories of global dimension 4.

For $G=\mathbb{Z} / 4$ we have that $H^{*}(\mathbb{Z} / 4, \mathbb{Z}) \cong \mathbb{Z}[u] / 4 u$ with $|u|=2$ and that the non trivial automorphism of $\mathbb{Z} / 4$ maps $u$ to $-u$; therefore $H^{4}(\mathbb{Z} / 4, \mathbb{Z}) / A u t(\mathbb{Z} / 4)=$ $\left\langle u^{2}\right\rangle=\mathbb{Z} / 4$.

For $G=(\mathbb{Z} / 2)^{2}$ we have that

$$
H^{4}\left((\mathbb{Z} / 2)^{2}, \mathbb{Z}\right) \cong \operatorname{ker}\left(S q^{1}: H^{4}\left((\mathbb{Z} / 2)^{2}, \mathbb{F}_{2}\right) \rightarrow H^{5}\left((\mathbb{Z} / 2)^{2}, \mathbb{F}_{2}\right)\right)=\left\langle x^{4}, x^{2} y^{2}, y^{4}\right\rangle
$$

where $H^{*}\left((\mathbb{Z} / 2)^{2}, \mathbb{F}_{2}\right)=\mathbb{F}_{2}[x, y]$ and $S q^{1}$ is the Steenrod operation, and up to automorphisms of $(\mathbb{Z} / 2)^{2}$ we get

$$
H^{4}\left((\mathbb{Z} / 2)^{2}, \mathbb{Z}\right) / \operatorname{Aut}\left((\mathbb{Z} / 2)^{2}\right)=\left\{\begin{array}{l}
0 \\
\left(x^{4}\right)=\left\{x^{4}, y^{4}, x^{4}+y^{4}\right\} \\
\left(x^{2} y^{2}\right)=\left\{x^{2} y^{2}, x^{2} y^{2}+x^{4}, x^{2} y^{2}+y^{4}\right\} \\
\left(x^{4}+x^{2} y^{2}+y^{4}\right)=\left\{x^{4}+x^{2} y^{2}+y^{4}\right\} .
\end{array}\right.
$$


Since we have a clear description for a base of $H^{4}\left((\mathbb{Z} / 2)^{2}, \mathbb{Z}\right)$, we will abuse the notation and denote with the symbols of $H^{4}\left((\mathbb{Z} / 2)^{2}, \mathbb{Z}\right)$ the elements of $H^{3}\left((\mathbb{Z} / 2)^{2}, \mathbb{C}^{*}\right)$. With this clarification the relevant terms of the second page of the LHS spectral sequence of the extension $1 \rightarrow \mathbb{Z} / 2 \rightarrow \mathbb{Z} / 4 \rightarrow \mathbb{Z} / 2 \rightarrow 1$ become

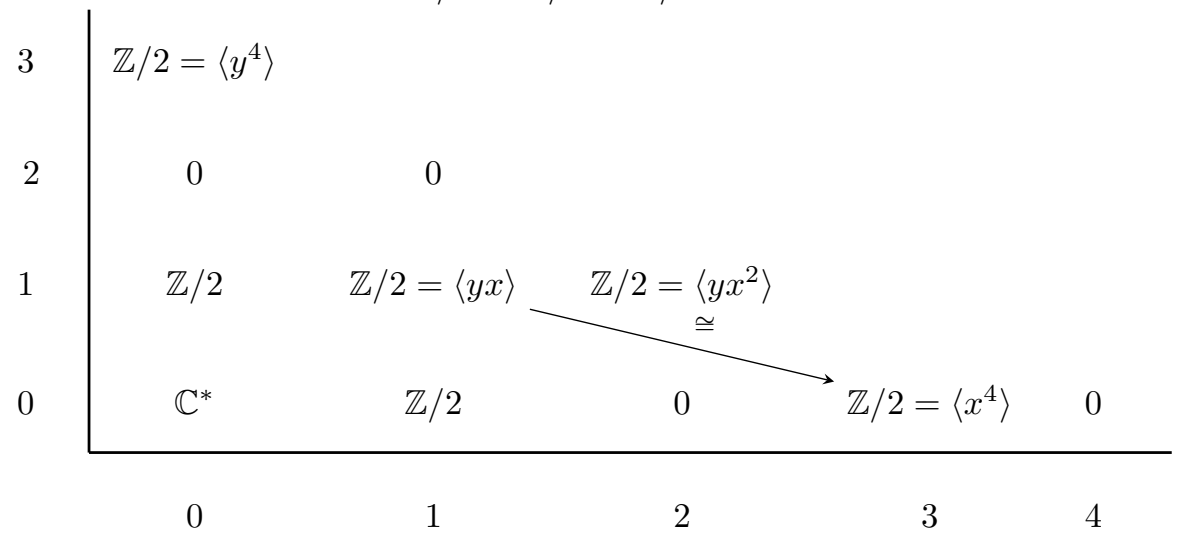

where the second differential is defined by the assignment $d_{2}\left(y x^{k}\right)=S q^{1}\left(x^{k+2}\right)$ with the class $x^{2}$ classifying the extension. We conclude that the only weak Morita equivalence that appears, which does not come from an automorphism of a group, is

$$
\operatorname{Vect}(\mathbb{Z} / 4,0) \simeq \operatorname{Vect}\left((\mathbb{Z} / 2)^{2}, x^{2} y^{2}\right)
$$

Therefore we see that there are exactly seven weak Morita equivalence classes of pointed fusion categories of global dimension 4 , namely the three for $\mathbb{Z} / 4$ :

$$
V \operatorname{ect}\left(\mathbb{Z} / 4, u^{2}\right), V e c t\left(\mathbb{Z} / 4,2 u^{2}\right), V \operatorname{ect}\left(\mathbb{Z} / 4,3 u^{2}\right)
$$

the three for $(\mathbb{Z} / 2)^{2}$ :

$$
V \operatorname{ect}\left((\mathbb{Z} / 2)^{2}, 0\right), V \operatorname{ect}\left((\mathbb{Z} / 2)^{2}, x^{4}\right), V \operatorname{ect}\left((\mathbb{Z} / 2)^{2}, x^{4}+y^{4}+x^{2} y^{2}\right)
$$

and the one that we have just constructed

$$
\operatorname{Vect}(\mathbb{Z} / 4,0) \simeq_{M} \operatorname{Vect}\left((\mathbb{Z} / 2)^{2}, x^{2} y^{2}\right)
$$

4.2. Non trivial action of $\mathbb{Z} / 2$ on $\mathbb{Z} / 4$. Consider the non trivial action of $\mathbb{Z} / 2$ on $\mathbb{Z} / 4$ and the abelian extension $1 \rightarrow \mathbb{Z} / 4 \rightarrow G \rightarrow \mathbb{Z} / 2 \rightarrow 1$. The group $G$ is either the Dihedral group $D_{8}$ in the case that the extension is a split extension or the quaternion group $Q_{8}$ in the case that the extension is a non-split extension.

In the case of $D_{8}$ the relevant elements of the second page of the LHS spectral sequence associated to the extension are: 

3 $\mathbb{Z} / 4=\langle a\rangle$
2
0
0
1
$\mathbb{Z} / 2=\langle e\rangle$
$\mathbb{Z} / 2=\langle b\rangle$
$\mathbb{Z} / 2$
0
$\mathbb{C}^{*}$
$\mathbb{Z} / 2$
0
$\mathbb{Z} / 2=\langle c\rangle \quad 0$
0
1

2

3
4

and they all survive to the page at infinity. Since $H^{3}\left(D_{8}, \mathbb{C}^{*}\right)=\mathbb{Z} / 4 \oplus \mathbb{Z} / 2 \oplus \mathbb{Z} / 2$ we may say that $H^{3}\left(D_{8}, \mathbb{C}^{*}\right) \cong\langle a\rangle \oplus\langle b\rangle \oplus\langle c\rangle$, and since $D_{8} \cong \mathbb{Z} / 4 \rtimes \mathbb{Z} / 2$ we have that $F=0$. The element $b \in H^{2}(\mathbb{Z} / 2, \mathbb{Z} / 4)$ defines the non trivial extension $Q_{8} \cong \mathbb{Z} / 2 \ltimes_{b} \mathbb{Z} / 4$.

The second page of the LHS spectral sequence of the extension $Q_{8} \cong \mathbb{Z} / 2 \ltimes_{b} \mathbb{Z} / 4$ becomes:

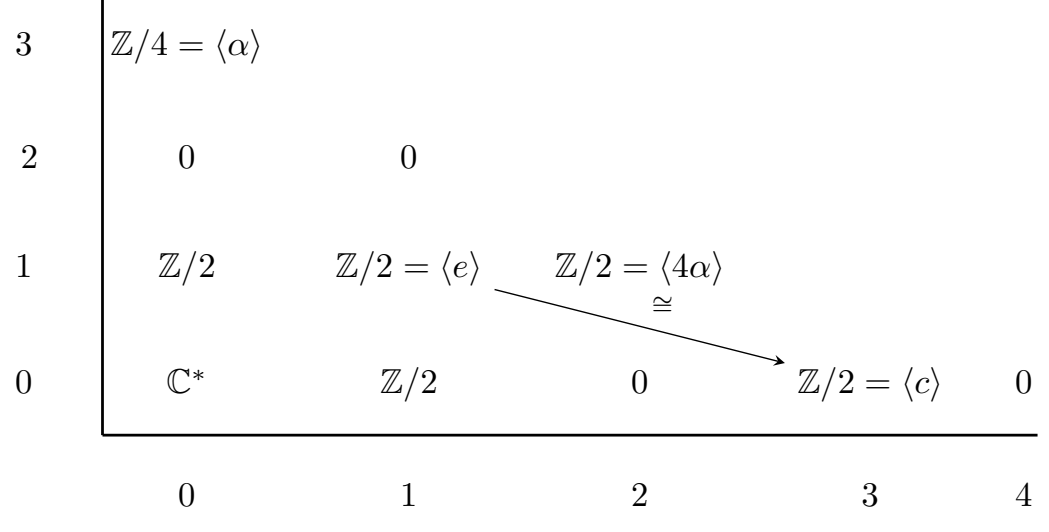

where $d_{2}: E_{2}^{1,1} \cong E_{2}^{3,0}$ is an isomorphism and $H^{3}\left(Q_{8}, \mathbb{C}^{*}\right)=\langle\alpha\rangle=\mathbb{Z} / 8$.

Therefore for these extensions we only have the weak Morita equivalences:

$$
\operatorname{Vect}\left(D_{8}, b\right) \simeq_{M} \operatorname{Vect}\left(Q_{8}, 0\right) \simeq_{M} \operatorname{Vect}\left(D_{8}, b \oplus c\right)
$$

where the equivalence of the right is obtained from the fact that $c$ does not survive the spectral sequence for the group $Q_{8}$, and the self Morita equivalence

$$
\operatorname{Vect}\left(Q_{8}, 4 \alpha\right) \simeq_{M} \operatorname{Vect}\left(Q_{8}, 4 \alpha\right)
$$

4.3. Extension of $\mathbb{Z} / 2 \times \mathbb{Z} / 2$ by $\mathbb{Z} / 2$. Consider the non-abelian extensions of the form $1 \rightarrow \mathbb{Z} / 2 \rightarrow G \rightarrow \mathbb{Z} / 2 \times \mathbb{Z} / 2 \rightarrow 1$, namely $D_{8}$ and $Q_{8}$.

The second page of the LHS spectral sequence for these extensions becomes: 


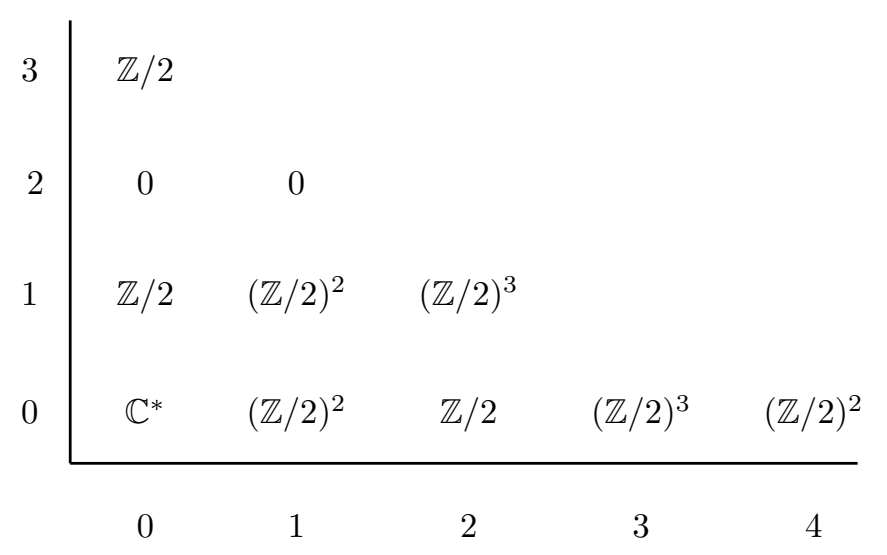

and we need only to concentrate in the differentials $d_{2}: E_{2}^{p, 1} \rightarrow E_{2}^{p+2,0}$ between the first two rows since we know that $E_{2}^{0,3}=\mathbb{Z} / 2$ survives the spectral sequence in all the groups.

First we will determine the differential $\bar{d}_{2}^{G}$ in the LHS spectral sequence for coefficients in the field of two elements $\mathbb{F}_{2}$. In this case

$$
E_{2} \cong H^{*}\left(\mathbb{Z} / 2 \times \mathbb{Z} / 2, \mathbb{F}_{2}\right) \otimes_{\mathbb{F}_{2}} H^{*}\left(\mathbb{Z} / 2, \mathbb{F}_{2}\right) \cong \mathbb{F}_{2}[x, y, e]
$$

and $\bar{d}_{2}^{G} e \in H^{2}\left(\mathbb{Z} / 2 \times \mathbb{Z} / 2, \mathbb{F}_{2}\right)$ represents the class that defines the extension $G$. It is known that the class $x^{2}+x y+y^{2}$ defines $Q_{8}$ [1, Lemma 2.10], the classes $x^{2}+x y, x y+y^{2}, x y$ define $D_{8}$ [1, pp. 130], and the classes $x^{2}, y^{2}, x^{2}+y^{2}$ define $\mathbb{Z} / 2 \times \mathbb{Z} / 4$.

Second we use the fact that for the group $(\mathbb{Z} / 2)^{2}$ we have the isomorphism

$$
H^{j}\left((\mathbb{Z} / 2)^{2}, \mathbb{Z}\right) \cong \operatorname{ker}\left(S q^{1}: H^{j}\left((\mathbb{Z} / 2)^{2}, \mathbb{Z} / 2\right) \rightarrow H^{j+1}\left((\mathbb{Z} / 2)^{2}, \mathbb{Z} / 2\right)\right)
$$

where $S q^{1}$ is the first Steenrod square. This implies that the map canonical map

$$
\left.H^{j}\left((\mathbb{Z} / 2)^{2}, \mathbb{Z} / 2\right)\right) \rightarrow H^{j}\left((\mathbb{Z} / 2)^{2}, \mathbb{C}^{*}\right)
$$

can be seen as the map

$$
\begin{gathered}
\left.H^{j}\left((\mathbb{Z} / 2)^{2}, \mathbb{Z} / 2\right)\right) \stackrel{S q^{1}}{\rightarrow} \\
k e r\left(S q^{1}: H^{j+1}\left((\mathbb{Z} / 2)^{2}, \mathbb{Z} / 2\right) \rightarrow H^{j+2}\left((\mathbb{Z} / 2)^{2}, \mathbb{Z} / 2\right)\right) \\
\cong H^{j+1}\left((\mathbb{Z} / 2)^{2}, \mathbb{Z}\right) \cong H^{j}\left((\mathbb{Z} / 2)^{2}, \mathbb{C}^{*}\right) .
\end{gathered}
$$

Therefore the second differential

$$
d_{2}^{G}: H^{p-2}\left((\mathbb{Z} / 2)^{2}, \mathbb{Z} / 2\right) \rightarrow H^{p}\left((\mathbb{Z} / 2)^{2}, \mathbb{C}^{*}\right)
$$

is isomorphic to the composite map

$$
\begin{aligned}
d_{2}^{G}: H^{p-2}\left((\mathbb{Z} / 2)^{2}, \mathbb{Z} / 2\right) & \rightarrow \operatorname{ker}\left(S q^{1}: H^{p+1}\left((\mathbb{Z} / 2)^{2}, \mathbb{Z} / 2\right) \rightarrow H^{p+2}\left((\mathbb{Z} / 2)^{2}, \mathbb{Z} / 2\right)\right) \\
& \cong H^{p+1}\left((\mathbb{Z} / 2)^{2}, \mathbb{Z}\right) \cong H^{p}\left((\mathbb{Z} / 2)^{2}, \mathbb{C}^{*}\right) \\
z & \mapsto S q^{1}\left(z \cup \bar{d}_{2}^{G} e\right) .
\end{aligned}
$$

Without loss of generality we may choose $\bar{d}_{2}^{G} e=x y+x^{2}$ for calculating the LHS spectral sequence for $D_{8}$. Applying the differential $d_{2}^{G}$ to the elements $1, x, y, x^{2}, x y, y^{2}$ we obtain that the surviving terms in the infinite page of the LHS spectral sequence for $D_{8}$ become: 

\begin{tabular}{l|l}
3 & $\mathbb{Z} / 2$ \\
& \\
&
\end{tabular}
$0 \quad 0$
$0 \quad \mathbb{Z} / 2=\langle e(y)\rangle \quad \mathbb{Z} / 2=\left\langle e\left(x y+x^{2}\right)\right\rangle$
$(\mathbb{Z} / 2)^{2}=\left\langle x^{2}, y^{2}\right\rangle$
0
$(\mathbb{Z} / 2)^{2}=\frac{\left\langle x^{4}, x^{2} y^{2}, y^{4}\right\rangle}{\left\langle x^{2} y^{2}+x^{4}\right\rangle} \quad 0$
$\mathbb{C}^{*}$
0
1
2
3
4

Here we are abusing the notation and we are using the explicit base of $H^{4}\left((\mathbb{Z} / 2)^{2}, \mathbb{Z}\right)$ to denote the elements in $H^{3}\left((\mathbb{Z} / 2)^{2}, \mathbb{C}^{*}\right)$. Since $E_{3}^{2,1}=\left\langle e\left(x y+x^{2}\right)\right\rangle$ we have that the weak Morita equivalences that we obtain in the extension are

$$
\begin{gathered}
V e c t\left(D_{8}, 0\right) \simeq_{M} \operatorname{Vect}\left((\mathbb{Z} / 2)^{3}, S q^{1}\left(e\left(x y+x^{2}\right)\right)\right) \\
V \operatorname{ect}\left(D_{8}, x^{4}\right) \simeq_{M} V \operatorname{Vect}\left((\mathbb{Z} / 2)^{3}, S q^{1}\left(e\left(x y+x^{2}\right)\right)+x^{4}\right) \\
V e c t\left(D_{8}, y^{4}\right) \simeq_{M} \operatorname{Vect}\left((\mathbb{Z} / 2)^{3}, S q^{1}\left(e\left(x y+x^{2}\right)\right)+y^{4}\right)
\end{gathered}
$$

and the self equivalence

$$
\operatorname{Vect}\left(D_{8}, e\left(x y+x^{2}\right) \simeq \operatorname{Vect}\left(D_{8}, e\left(x y+x^{2}\right) .\right.\right.
$$

The surviving terms for $Q_{8}$ with $\bar{d}_{2}^{G} e=x^{2}+x y+y^{2}$ are:

$$
\begin{aligned}
& 3 \mid \mathbb{Z} / 2 \\
& 200 \\
& 1 \quad 0 \quad \mathbb{Z} / 2=\left\langle e\left(x^{2}+x y+y^{2}\right)\right\rangle \\
& 0 \quad \begin{array}{llll}
\mathbb{C}^{*} & (\mathbb{Z} / 2)^{2}=\left\langle x^{2}, y^{2}\right\rangle & 0 & \mathbb{Z} / 2=\left\langle x^{2} y^{2}\right\rangle
\end{array} \\
& \begin{array}{ll}
0 & 1
\end{array}
\end{aligned}
$$

with $E_{\infty}^{0,3}=\mathbb{Z} / 2=\langle\alpha\rangle,\left\langle x^{2}+x y+y^{2}\right\rangle=\langle 2 \alpha\rangle$ and $\left\langle x^{2} y^{2}\right\rangle=\langle 4 \alpha\rangle$ where $\alpha$ is a generator $\langle\alpha\rangle=H^{3}\left(Q_{8}, \mathbb{C}^{*}\right)$ that was defined in section 44.2

Hence the only Morita equivalences that we obtain are

$$
\begin{gathered}
V e c t\left(Q_{8}, 0\right) \simeq \operatorname{Vect}\left((\mathbb{Z} / 2)^{3}, S q^{1}\left(e\left(x^{2}+x y+y^{2}\right)\right)\right) \\
V \operatorname{ect}\left(Q_{8}, 4 \alpha\right) \simeq V \operatorname{Vect}\left((\mathbb{Z} / 2)^{3}, S q^{1}\left(e\left(x^{2}+x y+y^{2}\right)\right)+x^{2} y^{2}\right)
\end{gathered}
$$

and the self Morita equivalences $V e c t\left(Q_{8}, 2 \alpha\right) \simeq_{M} V e c t\left(Q_{8}, 2 \alpha\right)$ and $V e c t\left(Q_{8}, 6 \alpha\right) \simeq_{M}$ $\operatorname{Vect}\left(Q_{8}, 6 \alpha\right)$.

Bundling up the previous results for the group $Q_{8}$ we obtain the following result: 
Proposition 4.1. Let us suppose that $\operatorname{Vect}\left(Q_{8}, k \alpha\right)$ is weakly Morita equivalent to $\operatorname{Vect}(G, \eta)$. Then

- For $k$ odd or $k=2,6, G$ must be isomorphic to $Q_{8}$ and $\eta$ must correspond to $j \alpha$ with $j$ odd or $j=2,6$.

- For $k=4, G$ must be isomorphic to $Q_{8}$ or $(\mathbb{Z} / 2)^{3}$.

- For $k=0, G$ must be isomorphic to $Q_{8}, D_{8}$ or $(\mathbb{Z} / 2)^{3}$.

Proof. First note that the action of $A u t\left(Q_{8}\right)$ on $H^{3}\left(Q_{8}, \mathbb{C}^{*}\right)$ is trivial. Second note that the only normal subgroups of $Q_{8}$ are its center and the cyclic ones generated by roots of unity and that they all fit into the central extension $1 \rightarrow \mathbb{Z} / 2 \rightarrow$ $Q_{8} \rightarrow(\mathbb{Z} / 2)^{2} \rightarrow 1$ or the non-split extension $1 \rightarrow \mathbb{Z} / 4 \rightarrow Q_{8} \rightarrow \mathbb{Z} / 2 \rightarrow 1$ that we have studied before. Since any weak Morita equivalence between pointed fusion categories comes from a normal and abelian subgroup of $Q_{8}$, the classification that we have done before exhausts all possibilities. For $k$ odd we know that $k \alpha$ survives to the restriction to the center and to the cyclic subgroups isomorphic to $\mathbb{Z} / 4$ and therefore $G$ can only be $Q_{8}$. The classes $2 \alpha$ and $6 \alpha$ trivialize on the center of $Q_{8}$ but these classes define extensions of $(\mathbb{Z} / 2)^{2}$ by $\mathbb{Z} / 2$ which are isomorphic to $Q_{8}$ and define cohomology classes which are precisely $2 \alpha$ and $6 \alpha$. The class $4 \alpha$ trivializes in all normal and abelian subgroups; in the case of the subgroup $\mathbb{Z} / 4$ the only group that may appear is $Q_{8}$, and in the case of the center we may obtain the weak Morita equivalence

$$
V e c t\left(Q_{8}, 4 \alpha\right) \simeq \operatorname{Vect}\left((\mathbb{Z} / 2)^{3}, S q^{1}\left(e\left(x^{2}+x y+y^{2}\right)\right)+x^{2} y^{2}\right) .
$$

Finally, the trivial class produces only the group $D_{8}$ in the case of the subgroup $\mathbb{Z} / 4$ and $(\mathbb{Z} / 2)^{3}$ in the case of the center; some weak Morita equivalences are

$$
\operatorname{Vect}\left(Q_{8}, 0\right) \simeq \operatorname{Vect}\left((\mathbb{Z} / 2)^{3}, S q^{1}\left(e\left(x^{2}+x y+y^{2}\right)\right)\right) \simeq_{M} \operatorname{Vect}\left(D_{8}, b\right) .
$$

\section{REFERENCES}

[1] A. Adem and R. J. Milgram. Cohomology of finite groups, volume 309 of Grundlehren der Mathematischen Wissenschaften [Fundamental Principles of Mathematical Sciences]. Springer-Verlag, Berlin, 1994.

[2] B. Bakalov and A. Kirillov, Jr. Lectures on tensor categories and modular functors, volume 21 of University Lecture Series. American Mathematical Society, Providence, RI, 2001.

[3] A. A. Davydov. Finite groups with the same character tables, Drinfel'd algebras and Galois algebras. In Algebra (Moscow, 1998), pages 99-111. de Gruyter, Berlin, 2000.

[4] R. Dijkgraaf, V. Pasquier, and P. Roche. Quasi Hopf algebras, group cohomology and orbifold models. Nuclear Phys. B Proc. Suppl., 18B:60-72 (1991), 1990. Recent advances in field theory (Annecy-le-Vieux, 1990).

[5] P. Etingof, D. Nikshych, and V. Ostrik. On fusion categories. Ann. of Math. (2), 162(2):581-642, 2005.

[6] P. Etingof, D. Nikshych, and V. Ostrik. Weakly group-theoretical and solvable fusion categories. Adv. Math., 226(1):176-205, 2011.

[7] L. Evens. The cohomology of groups. Oxford Mathematical Monographs. The Clarendon Press, Oxford University Press, New York, 1991. Oxford Science Publications. 
[8] M. V. Movshev. Twisting in group algebras of finite groups. Funktsional. Anal. $i$ Prilozhen., 27(4):17-23, 95, 1993.

[9] M. Müger. From subfactors to categories and topology. I. Frobenius algebras in and Morita equivalence of tensor categories. J. Pure Appl. Algebra, 180(12):81-157, 2003.

[10] D. Naidu. Categorical Morita equivalence for group-theoretical categories. Comm. Algebra, 35(11):3544-3565, 2007.

[11] V. Ostrik. Module categories over the Drinfeld double of a finite group. Int. Math. Res. Not., (27):1507-1520, 2003.

[12] V. Ostrik. Module categories, weak Hopf algebras and modular invariants. Transform. Groups, 8(2):177-206, 2003.

Departamento de Matemáticas y Estadística, Universidad del Norte, Km.5 Vía Antigua a Puerto Colombia, Barranquilla, Colombia.

E-mail address: bjongbloed@uninorte.edu.co 